\title{
Resonant Tunneling Diodes: Models and Properties
}

\author{
JIAN PING SUN, GEORGE I. HADDAD, LIFE FELLOW, IEEE, \\ PINAKI MAZUMDER, SENIOR MEMBER, IEEE, AND JOEL N. SCHULMAN
}

\begin{abstract}
The resonant tunneling diode (RTD) has been widely studied because of its importance in the field of nanoelectronic science and technology and its potential applications in very high speed/functionality devices and circuits. Even though much progress has been made in this regard, additional work is needed to realize the full potential of RTD's. As research on RTD's continues, we will try in this tutorial review to provide the reader with an overall and succinct picture of where we stand in this exciting field of research and to address the following questions: What makes RTD's so attractive? To what extent can RTD's be modeled for design purposes? What are the required and achievable device properties in terms of digital logic applications? To address these issues, we review the device operational principles, various modeling approaches, and major device properties. Comparisons among the various RTD physical models and major features of RTD's, resonant interband tunneling diodes, and Esaki tunnel diodes are presented. The tutorial and analysis provided in this paper may help the reader in becoming familiar with current research efforts, as well as to examine the important aspects in further RTD developments and their circuit applications.
\end{abstract}

Keywords-Nanoelectronics, quantum theory, quantum wells, resonant tunneling devices, semiconductor device modeling.

\section{INTRODUCTION}

Over the past two decades, resonant tunneling diodes (RTD's) have received a great deal of attention following the pioneering work by Esaki and Tsu [1]. Significant accomplishments have been achieved in terms of RTD device physics, modeling, fabrication technology, and circuit design and applications. The RTD has been widely studied, and well over a thousand research papers have been written on various aspects of this seemingly simple device. Yet, whether RTD's will find their way into mainstream

Manuscript received March 11, 1997; revised December 22, 1997. This work was supported by the ARO-URI program under Grant DAAL03-92G-0109.

J. P. Sun, G. I. Haddad, and P. Mazumder are with the Center for High Frequency Microelectronics, Solid State Electronics Laboratory, Department of Electrical Engineering and Computer Science, University of Michigan, Ann Arbor, MI 48109-2122 USA.

J. N. Schulman was with the Center for Collaborative Research, University of Tokyo, Tokyo, Japan. He is now with HRL Laboratories, Malibu, CA 90265 USA.

Publisher Item Identifier S 0018-9219(98)02492-X. electronics in the future remains inconclusive. The research is ongoing and, in some areas, very active. Why has this device, typically consisting of two potential barriers and one quantum well, been so attractive to the electronics research community for such a long period? With all the knowledge about RTD's so far acquired, will RTD's become practical? What are the main issues of current research on RTD's? To get at these questions, we present in this tutorial review an updated and succinct picture that addresses some important aspects of RTD's. For formal treatments and detailed analysis on specific topics of RTD's, the interested reader can find a number of excellent papers, review articles, and books, which will be referred to in the relevant context. This paper will cover the device aspects, while the circuit aspects on RTD circuit design and digital applications will be reviewed in an accompanying paper in this issue [2].

It is well documented that today's advanced information technology is mainly attributed to the electronic representation and processing of information in a low-cost, highspeed, very compact, and highly reliable fashion, and that the quest and accomplishments of continual miniaturization and integration of solid-state electronics have been the key to the success of the computer industry and computer applications. The advanced multimedia infrastructure and services in the future will demand further reduction in chip size. Chip density, represented by memory technology, has been following Moore's law and has roughly doubled every other year over the last three decades. The trend remains strong and definite, at least for the foreseeable future. For example, a $0.15-\mu \mathrm{m}$ process technology has been implemented in the first 4-Gb dynamic random access memory (DRAM) unveiled in 1997, and the feature size of DRAM transistors is projected to be $0.18 \mu \mathrm{m}(1 \mathrm{~Gb})$ in $2001,0.13 \mu \mathrm{m}(4 \mathrm{~Gb})$ in $2004,0.10 \mu \mathrm{m}(16 \mathrm{~Gb})$ in 2007, and $0.07 \mu \mathrm{m}(64 \mathrm{~Gb})$ by 2010 [3]. A natural and realistic question, then, is whether this desired trend will continue indefinitely. While an ultimate limit on the downscaling of conventional transistors and integrated circuits (IC's) will eventually be reached, device physicists and 
IC engineers have pondered answers, both evolutionary and revolutionary, to the challenge. While the downscaling of conventional transistors enjoys an exceptional, rapid evolution, revolutionary device concepts have been actively sought, particularly in the two related areas known as nanoelectronics and single electronics [4]. For a more recent overview of nanoelectronic devices, see [5].

The idea of nanoelectronics was popularized in the mid1980 's, when pioneering work on resonant tunneling and bandgap engineering in low-dimensional semiconductor quantum wells and superlattices grew and was championed by several groups for the exploration of new opportunities for circumventing the limit on the downscaling of conventional transistors and IC's. The physics and electronics communities worldwide participated with enthusiastic research efforts, with rapid progress made in understanding mesoscopic transport physics as well as nanofabrication technology. The RTD, and its several variations, has become a research focus in nanoelectronics for its promise as a primary nanoelectronic device for both analog and digital applications.

It is well known that when the size of a system becomes comparable to the electron wavelength, quantum effects become dominant. This occurs when transistors are downscaled and their characteristic dimensions reach the nanometer range, leading to new phenomena and possible novel devices based on quantum tunneling mechanisms. For nanoelectronics to become a reality, it is essential that the new devices and circuits must be fabricated with nanometer precision, and one must be able accurately to design the devices and circuits. This has led to research efforts and accomplishments in three areas: nanofabrication, quantum modeling, and circuit innovations.

For device realization, nanofabrication technology has made impressive advances during the last decade by routinely producing artificial semiconductor structures using molecular-beam epitaxy, metal-organic chemical vapor deposition, and chemical-beam epitaxy. Accurately controlled feature sizes as small as monolayers of atoms in the growth direction for dissimilar semiconductor materials, or heterostructure systems, have been achieved. Nanoscale lithography and patterning by electron-beam lithography have also been highly developed in the direction perpendicular to the growth direction. Although further improvements in this area call for more precise control, better resolution, and improved interfaces, recent advances in nanofabrication technology have brought quantum effect device concepts to reality and have presented a great challenge for device physicists in the theoretical analysis of nanoelectronic devices. For a recent review of nanofabrication, the reader is referred to [6].

Continuing effort in quantum transport modeling of RTD's is motivated by the need to understand device operation and to provide a primary test for developing theoretical tools for nanoelectronic devices. Not surprisingly, this is very different from traditional device modeling. Moreover, it provides valuable knowledge of the quantum aspects of electron transport in mesoscopic systems. Since the useful device properties, e.g., fast switching operation between ON and OFF states, are a consequence of the desired and controlled electron motion in the device, it is essential for device designers to understand and quantify the transport processes. Based on physical models, one can adjust the structural parameters and suppress the unwanted processes through device design and optimization. For conventional devices such as metal-oxide-semiconductor (MOS) and bipolar transistors with feature sizes much larger than nanometers, semiclassical transport models have proven to be adequate for successful device downscaling development. These device models become invalid in the nanometer regime, however, since the classical transport concepts treat electrons (and holes) as particles, and the transport parameters are defined by taking ensemble averages of the particle motion. In nanostructures, a device theory that can properly treat quantum transport phenomena between macroscopic and microscopic scales is therefore needed. Work in this field has invoked mesoscopic physical principles and hierarchical quantum device models for formulating and quantifying nanoelectronic transport and device analysis. However, the available nanoelectronic device models have not been well established to the standards of conventional device models. For example, it is still a formidable task to accurately predict the I-V characteristics of nanoelectronic devices, such as RTD's. This topic is considered further in Section III.

Among the numerous nanoelectronic devices proposed and demonstrated, the RTD is perhaps the most promising candidate for digital circuit applications due to its negative differential resistance (NDR) characteristic, structural simplicity, relative ease of fabrication, inherent high speed, flexible design freedom, and versatile circuit functionality. There is a good practical reason to believe that RTD's may be the next device based on quantum confined heterostructures to make the transition from the world of research into practical application. Progress in epitaxial growth has improved the peak-to-valley current ratio at room temperature even beyond that required for many circuit applications. This temperature requirement is the single most important feature that any new technology must satisfy. It is what distinguishes the RTD from other interesting quantum device concepts that have been proposed but that show weak, if any, desired phenomena at room temperature. A variety of circuit functions has already been demonstrated, providing proof-of-concept of proposed applications [7]-[10]. The main issue at present is not, in fact, the RTD performance itself but the monolithic integration of RTD's with transistors [high electron mobility transistors (HEMT's) or heterojunction bipolar transistors (HBT's)] into integrated circuits with useful numbers and density of devices. Major challenges include the variation in the current-voltage characteristic of the RTD's across a wafer and from wafer to wafer, fabrication-dependent parasitic impedances, and edge effects as the RTD mesa area is decreased in order to reduce the intrinsic parasitic impedances and to achieve higher integration levels. Recently developed techniques for 


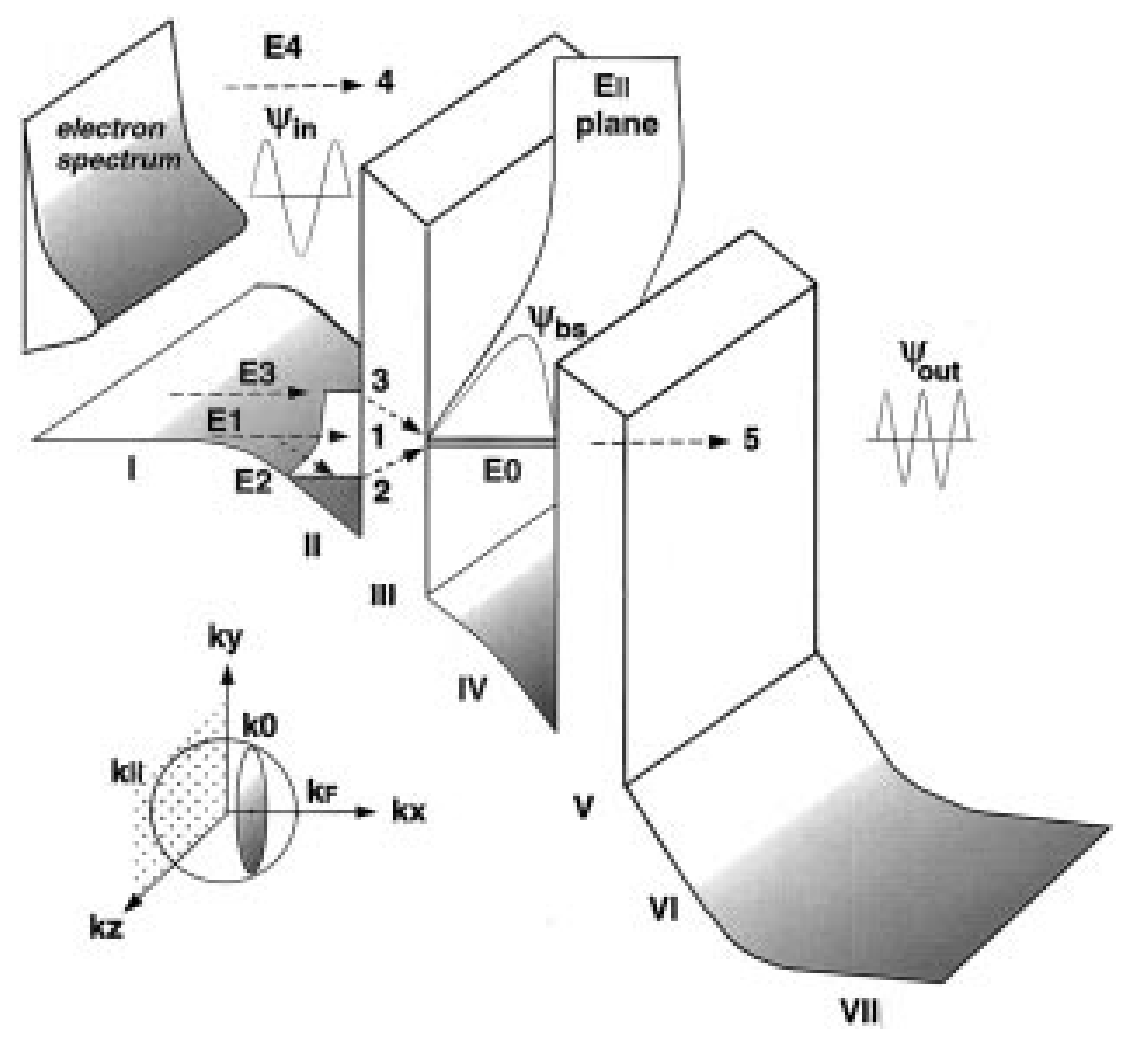

Fig. 1. Physical processes in double-barrier tunneling.

providing feedback during epitaxial growth via optical and photoemission probes have greatly improved the situation as far as uniformity of growth is concerned. It is for these reasons that RTD research has been sustained for more than two decades and may now be rapidly approaching the stage of technology implementation. A more specific account of RTD device features and properties as compared with other NDR quantum devices is given in Section IV.

This paper is organized as follows. The resonant tunneling phenomena is first reviewed in Section II, where a simple physical picture of the origin of the NDR is given for a basic understanding of RTD operation. Section III deals with theoretical models of RTD's. The hierarchical models of RTD's with various levels of sophistication are compared and recent progress is summarized. Although RTD models have inspired intriguing physical and mathematical formulations, it is not the purpose of this paper to go into a detailed treatment of quantum transport. Only the conceptual framework and main features of various formalisms are examined, without engaging in details of the theory. For purposes of illustration, however, RTD operational principles and modeling issues are exemplified using the simple envelope function formulation. Section IV, on the analysis of RTD properties, discusses the considerations for desirable RTD characteristics for digital circuits and related issues in terms of carrier transport restrictions and realistic material and structural considerations. The main features of various RTD's, resonant interband tunnel diodes (RITD's), and Esaki TD's are also compared and summarized. In the final section, concluding remarks are given highlighting the accomplishments and existing concerns in RTD research. This tutorial review on RTD issues cannot be encyclopedic, with an obvious omission of the high-frequency aspects of RTD's, but it is hoped that an overall picture of where we are with an updated account of this exciting research frontier is presented.

\section{RESONANT TUNNELING}

\section{A. Double-Barrier Quantum Well (DBQW) Structure}

The basic RTD device configuration is a DBQW structure of nanometer dimensions, including two contacts as depicted in Fig. 1, where the regions I, II and VI, VII are heavily doped contacts made from a semiconductor with a relatively small bandgap, e.g., GaAs. These layers comprise the emitter and collector, respectively. Regions III and V are quantum barriers made from a semiconductor with a relatively larger bandgap, e.g., AlGaAs, and in particular a positive conduction-band offset with respect to the smaller bandgap semiconductor. Region IV between the two barriers is the quantum well made again from the smaller bandgap semiconductor. It is sometimes also called the base, whether or not electrical contact is actually being attempted. The structure is shown in terms of electron energy versus distance under bias because we are interested in the electron transport process, which is essentially the electron motion within a certain energy-band structure under applied bias voltages. Because the characteristic dimensions of the DBQW structure are comparable with the electron wavelengths, the wave nature of electrons leads to 
quantum phenomena such as interference, tunneling, energy quantization, etc. As a result, resonant tunneling phenomena occur in DBQW structures and form the basis for RTD operation.

The basic RTD structure shown in Fig. 1 may have many variations in terms of the potential (energy) profile, which is determined primarily by the specific material system used. We will consider issues of the material system and device structure in Section IV when we discuss device properties. Fig. 1 gives a descriptive picture of the major physical processes pertaining to resonant tunneling and current transport components in RTD's.

\section{B. Physical Processes in RTD's}

Envision a spectrum of electrons in region I, driven by a bias voltage applied across the RTD contacts, incident upon the DBQW structure shown in Fig. 1. The electron spectrum is considered to be distributed in energy according to the Fermi-Dirac statistics as the contacts are typically heavily doped to provide a low ohmic contact and high current density. The use of the Fermi distribution implies that the electrons in region I are assumed in thermal equilibrium due to the interactions among the electrons and their surroundings in contact region I. The same assumption is made for contact region VII. Electrons are also incident from the right-hand-side contact region but are not shown in the figure; the current due to them is small under the bias condition as shown.

A first look at the RTD structure brings out the general features of the electron wave as it traverses the DBQW, resulting in the main characteristic of RTD's, namely, the NDR. The electron undergoes multiple reflections from the interfaces due to its wave nature, leading to the resonant nature of the tunneling. Also, there are dimensional changes across the interfaces, i.e., the three-dimensional (3-D) density of states in the emitter versus the twodimensional (2-D) density of states in the quantum well, as described in the next section. The multiple reflections cause destructive or constructive interference depending on the wavelength of the particular electron. For electrons with specific wavelengths that favor constructive interference, a near-unity transmission probability may be found at energies corresponding to these wavelengths. In other words, resonant tunneling occurs at these resonant energy levels, which may have a finite width in energy due to broadening mechanisms. This process is indicated in Fig. 1 by process 1 for an electron with energy $E_{1}$ coincident with the resonant energy $E_{0}$. In addition to the transport through the resonant levels, other processes can also be identified. In process 2 , an electron is first scattered into an energy level $E_{2}$ in the emitter accumulation layer, which is a 2-D potential well in region II. It may then absorb a phonon and sequentially tunnel through the resonant level $E_{0}$. Similarly, in process 3 , an electron with an initial energy $E_{3}$ may interact with the lattice vibration by emitting a phonon, then tunnel through $E_{0}$. On the other hand, electrons with sufficiently high energies (for example, $E_{4}$ ) may overcome the barriers by thermionic emission, as indicated by process
4. Note that the incident electrons also have a finite, but small, probability to tunnel through the nonresonant energy ranges that lie between the resonances. This current is an important contribution to the valley current.

The physical processes involved in RTD operation are in actuality much more complex than the preceding simple description and are especially complicated by the electron's interaction with its environment. First of all, the electrons have particle and energy exchanges with the battery applying the bias voltage, which makes the device open to the outside environment and very different from an isolated quantum system. Moreover, the electrons in the RTD structure have interactions with lattice vibration, impurities, interface roughness, and alloy disorder, as well as the interaction among themselves. In the wave picture, an incident electron wave is scattered by not only the heterostructure potential profile but also the scattering potentials originating from these other scatterers. These processes may affect the device properties significantly, making an accurate physical model of realistic RTD's a formidable task.

\section{Origin of Negative Differential Resistance}

An important feature of resonant tunneling is that electrons are incident from the 3-D contacts into the 2-D quantum well, where the electrons have a plane-wavelike motion only in the $y$ and $z$ directions, as shown in Fig. 1. We designate the in-plane wave vector or transverse momentum $k_{\|}=\sqrt{k_{y}^{2}+k_{z}^{2}}$. Following Luryi's explanation [11], the conservation of the crystal transverse momentum of the tunneling electron is responsible for the NDR.

The electron energy in the 3-D emitter contact can be written as

$$
E_{3 D}=E_{C}+\frac{\hbar^{2} k_{x}^{2}}{2 m^{*}}+\frac{\hbar^{2} k_{\|}^{2}}{2 m^{*}}
$$

where $E_{C}$ is the bottom of the conduction-band in the emitter in region I and the effective masses in the different layers are assumed to be the same at this point.

The electron energy in the quantum well is given by

$$
E_{2 D}=E_{n}+\frac{\hbar^{2} k_{\|}^{2}}{2 m^{*}}
$$

where $E_{n}$ is the energy of an available $n$th subband in the quantum well. During tunneling, a reduction of dimensionality occurs, but the tunneling electrons do not see any potential change in the transverse directions and their transverse momentum is therefore conserved. The electron longitudinal momentum $k_{x}$ however, generally changes with distance except in the flat band-edge regions. As $E_{0}(n=0)$ is an accessible unoccupied state in the quantum well, from energy conservation one sees that tunneling is possible only for electrons with their momenta in a disk with $k_{x}=k_{0}$ in the emitter Fermi sphere, where $k_{0}^{2}=2 m^{*}\left(E_{0}-E_{C}\right) / \hbar^{2}$. As the emitter-base potential rises under bias, so does the number of electrons that can tunnel. For $E_{0}=E_{C}$, which corresponds to $k_{0}=0$, 
the number of tunneling electrons per unit area reaches a maximum. When $E_{C}$ rises above $E_{0}$, then at $T=0 \mathrm{~K}$, there are no electrons in the emitter that can tunnel into the quantum well while conserving their transverse momentum. The tunneling current density therefore has a sharp drop from its maximum value. Further increase of the bias and/or temperature will further lift up the electron distribution in the emitter, and electron thermionic emission and tunneling through the top regions of the barriers will lead to an increase in current density. It can then be appreciated that the origin of the NDR in the RTD characteristic requires transverse momentum conservation as a condition.

\section{RTD DEVICE MODELS}

Formal treatment of the modeling of RTD's entails advanced quantum transport theory, possibly including rather intricate formulations of multiband effective mass theory, quantum statistical theory based on the density matrix, Wigner functions, and Green's functions. Frensley, Ferry, Grubin, and other authors have written extensive review papers on related subjects, and interested readers can find more detailed information from these reviews [12], [13]. We present in this section an overview of various RTD models in a simplified conceptual framework of quantum transport and a tabulated summary of the main features and differences of the models. The RTD's dc I-V characteristic is taken as the primary example for illustrating RTD modeling issues.

Significant effort has been devoted to developing physical models of RTD's with varying degrees of sophistication and success. The interest in achieving an accurate RTD model lies in the fundamental motivation to gain insight into mesoscopic transport and provide guidance for optimal device design, which are indispensable for conceiving new devices and pursuing realistic device development. Moreover, we believe that there exists a lag in theoretical modeling of quantum transport as compared to nanofabrication technology. Quantum device modeling has yet to be as well established as modeling capabilities for conventional MOS field-effect transistors (FET's) and bipolar transistors. On the other hand, quantum transport problems in nanoelectronic devices and their complicated material and structural dependence demand heavy reliance on computer simulation tools. In that sense, RTD models serve as a test vehicle for device modeling capabilities as we progress into the nanoelectronic regime.

Device terminal properties are determined by studying carrier transport of the electrons, whose available energies are described by the band structure. The carrier motion should include the major physical processes, such as those depicted in Fig. 1, in a specific band profile. In order to compute RTD I-V characteristics, it is clear that RTD modeling should include two important aspects.

1) Proper energy-band parameters for a particular RTD structure, built up from dissimilar materials and their interfaces. For example, the band offsets at the heterointerfaces, carrier effective masses, and other ma-

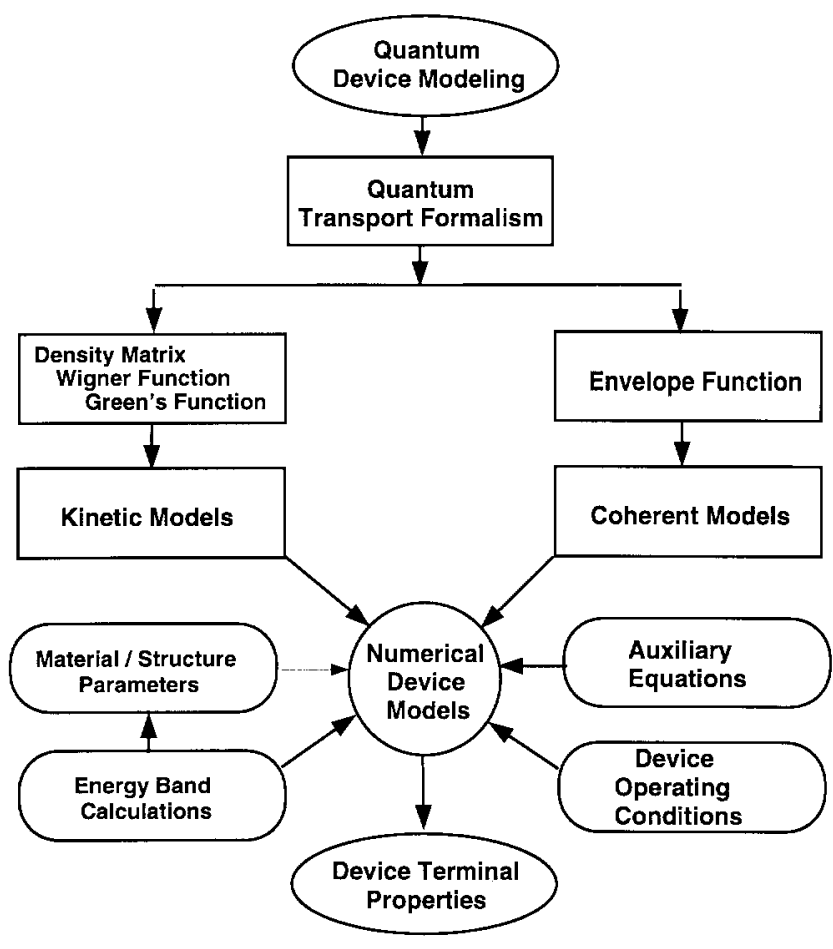

Fig. 2. Elements of quantum device modeling.

terial parameters derived from the band structure must be available with accuracy.

2) A proper transport formalism, which must be able to model the major processes in the RTD. Since simplifications, approximations, and numerical discretization are always used in the various models, they must not violate the basic physical laws and principles of quantum mechanics. However, in practice, these simplifications and approximations often compromise some of these principles.

The general elements of quantum device models are outlined in Fig. 2, which will be discussed in some detail.

From a first-principles point of view, the RTD as a nanoelectronic device is in general an open quantum system, in which electron transport is three dimensional, time dependent, time irreversible, dissipative, and many-body interactive, with both particle and energy exchanges with the device's environment. The device system is therefore very different from a simple isolated quantum system, where a conservative Hamiltonian and the boundary conditions for the Schrödinger equation may be readily formulated. On the other hand, since a full many-body formalism that is computationally manageable for such open device systems has not been well established, and may not be necessary for calculations of specific device properties, approximations and simplifications are typically used in various modeling approaches to simplify the calculations, provided that the major transport processes can be properly modeled. Recent efforts in RTD modeling have generated fruitful results for evaluation of quantum effects in RTD's and for device design. In terms of the electron state description and the 
capability of correctly treating the electron interactions with its environment, the existing RTD physical models can be classified into two categories: dynamic or coherent models and kinetic models. We first indicate their common ingredients before identifying their differences.

According to quantum mechanics, an electron can be described by its quantum state, represented by its state function. A simple or pure electron state is generally represented by the electron wave function and may be specified by its amplitude, momentum (wave vector), energy, and phase; its time evolution follows the time-dependent Schrödinger equation. An interaction between an electron and an optical phonon, however, may break the electron's phase coherence and generate correlations with other microparticles. We then speak of incoherent interactions and mixed electronic states. This is because the interacting particles have no phase relation with the incident electron wave, and it is therefore in practice impossible to trace the variation of the electron wave function of every particle. According to quantum statistical theory, the mixed state may be characterized by the density matrix, Wigner (distribution) functions, or Green's functions. For device modeling purposes, we may have the view that common to all quantum transport models, one takes a model function to represent electron states in a quantum device. Once the model function is evaluated for a specific device under specific operating conditions, other physical quantities of interest as well as device properties can all be calculated from the available model function. It is the chosen model function that makes major differences in terms of the formalism, capabilities, and results from one model to another. The model function in the coherent dynamic models is typically the envelope function, whereas in the kinetic models, the density matrix, Wigner function, and Green's functions are employed and formulated. These functions representing electron states in quantum devices are capable of modeling, to varying degrees, the physical processes in the device, such as electron transmission, reflection, and scattering processes, in a unified formulation. The central formulation in a model can be a single equation or a set of equations, depending on the chosen model. Since the approximations often render an approach inadequate for all the processes to be modeled even approximately, however, additional formulation may still be needed. The central equation is typically a nonlinear differential or differentialintegral equation, and solutions to the equation require extensive numerical work and computer programming as well as significant computer resources.

An important issue closely related to the central equations is boundary conditions. It is essential to have a proper treatment of boundary conditions for any device model. It is especially critical for quantum device models where the device system is open to particle reservoirs and driven far from equilibrium. As restricted by the scope of this paper, however, we will only point out the boundary conditions in RTD models. For a comprehensive review on this topic, the reader is referred to an in-depth analysis by Frensley [12].

As in any physical system, one would like to find how a system evolves in time with a computable equation of motion. Device transient and high-frequency properties, such as switching times and device admittance (impedance) at high frequencies, require a time-dependent solution of the model function. For device dc I-V characteristics, solutions of the time-independent or steady-state equation of the model function often suffice. In this paper, we identify in each approach a central model equation associated with its model function. A solution for the model function leads to the device terminal properties, such as I-V characteristics.

\section{A. Envelope Function Models of RTD's}

RTD models based on the envelope function description of electron states have been popular and very useful in studying RTD's and other nanoelectronic devices because they are conceptually simple, computationally efficient, and capable of modeling important properties of RTD's. We will outline RTD models based on the envelope function in this section to illustrate the main issues.

The envelope function description for electron states in semiconductors has been well documented [14] and is familiar to device engineers. It is based on effective mass theory, where the internal crystal potential is integrated into the effective response of carriers to an external applied force, greatly simplifying the treatment of carrier transport in semiconductor devices. For RTD models, since quantum phenomena must be included, the effective mass Schrödinger equation is invoked to calculate the electron state function, or the envelope wave function, neglecting the rapidly varying periodic component. This is the first major approximation made in the envelope function formalism, which brings about a great simplification, at the price of ignoring the microscopic information of the central cell part of the true wave function. Another major approximation involved in the envelope function approach is that it is limited to pure state calculations, to be discussed shortly.

The time-independent effective mass Schrödinger equation is the central equation of the envelope function model

$$
\left[-\frac{\hbar^{2}}{2} \nabla\left(\frac{1}{m^{*}} \nabla\right)+V(\mathbf{r})\right] \psi_{n}(\mathbf{r})=\left[E(\mathbf{k})-E_{n}(0)\right] \psi_{n}(\mathbf{r})
$$

where the potential term $V(\mathrm{r})$ includes the device energyband offset for the heterojunction, the bias voltage applied across the device, the contributions from the doping impurities, and mobile electronic charge. These enter $V(\mathbf{r})$ in a mean-field, self-consistent fashion (the Hartree potential) through the coupling of (3) with Poisson's equation. Additionally, the electron correlation and exchange potential due to the interaction of electrons may also be included. The effective mass $m^{*}$ is specified according to the corresponding materials. The subscript $n$ indicates the $n$th band, $\mathbf{k}$ indicates the electron wave vector, and $\mathbf{r}$ indicates the position vector. Equation (3) is often converted in practice into a one-dimensional (1-D) calculation using a weighting function $W(\mathbf{k})$, also known as the supply function for incident electrons, which effectively integrates over transverse wave vectors $k_{\|}$. The Schrödinger equation 
Table 1 Features of Various RTD Models

\begin{tabular}{|c|c|c|c|c|}
\hline Model type & Coherent & & Kinetic & \\
\hline $\begin{array}{l}\text { Model } \\
\text { functions }\end{array}$ & $\begin{array}{l}\text { envelope } \\
\text { function }\end{array}$ & $\begin{array}{l}\text { density } \\
\text { matrix }\end{array}$ & $\begin{array}{l}\text { Wigner } \\
\text { function }\end{array}$ & $\begin{array}{l}\text { Green's } \\
\text { functions }\end{array}$ \\
\hline $\begin{array}{l}\text { Central } \\
\text { equation }\end{array}$ & $\begin{array}{l}\text { effective mass } \\
\text { cquation }\end{array}$ & $\begin{array}{l}\text { Lionville } \\
\text { equations }\end{array}$ & $\begin{array}{l}\text { Liouville } \\
\text { equation }\end{array}$ & $\begin{array}{l}\text { Dyson } \\
\text { cquation }\end{array}$ \\
\hline Formalism & $\begin{array}{l}\text { pure } \\
\text { state }\end{array}$ & $\begin{array}{l}\text { mixed } \\
\text { state }\end{array}$ & $\begin{array}{l}\text { mined } \\
\text { stable }\end{array}$ & $\begin{array}{l}\text { mixed } \\
\text { stiate }\end{array}$ \\
\hline $\begin{array}{l}\text { Boundary } \\
\text { conditions }\end{array}$ & QTBM & QTBM & $\begin{array}{l}\text { injected distribution } \\
\text { function }\end{array}$ & QTBM \\
\hline $\begin{array}{l}\text { Phonorl } \\
\text { scattering }\end{array}$ & $\begin{array}{l}\text { various } \\
\text { approximnations }\end{array}$ & $\begin{array}{l}\text { relaxation } \\
\text { approximation }\end{array}$ & $\begin{array}{l}\text { relaxation } \\
\text { approxinnation }\end{array}$ & $\begin{array}{l}\text { self energy } \\
\text { treat ment }\end{array}$ \\
\hline $\begin{array}{l}\text { Impurity } \\
\text { scattering }\end{array}$ & $y e s$ & ro report & no report & yes \\
\hline $\begin{array}{l}\text { Nlloy } \\
\text { scattering }\end{array}$ & yes & no report & no report & yes \\
\hline $\begin{array}{l}\text { Interface } \\
\text { scattering }\end{array}$ & yes & no repori & no report & yes \\
\hline $\begin{array}{l}\text { Hartree Self- } \\
\text { consistency }\end{array}$ & yes & yes & yes & yes \\
\hline $\begin{array}{l}\text { Emitter } \\
\text { quantization }\end{array}$ & yes & yes & yes & yes \\
\hline $\begin{array}{l}\text { Treatment of } \\
\text { scattering }\end{array}$ & $\begin{array}{l}\text { varions } \\
\text { approximnations }\end{array}$ & $\begin{array}{l}\text { relaxation } \\
\text { approximation }\end{array}$ & $\begin{array}{l}\text { relaxation } \\
\text { approximation }\end{array}$ & self-energy \\
\hline $\begin{array}{l}\text { Multiband } \\
\text { effects }\end{array}$ & yes & no report & no report & yes \\
\hline $\begin{array}{l}\text { Quantum } \\
\text { dissipation }\end{array}$ & $\mathrm{n} O \mathrm{O}$ & yos & yes & yes \\
\hline $\begin{array}{l}\text { Transient } \\
\text { modeling }\end{array}$ & yes & yes & yes & in progress \\
\hline $\begin{array}{l}\text { Figh-frequency } \\
\text { models }\end{array}$ & yes & yes & yes & in progres: \\
\hline $\begin{array}{l}\text { I-V characteristic } \\
\text { prediction }\end{array}$ & $\begin{array}{l}\text { not } \\
\text { consistent }\end{array}$ & $\begin{array}{l}\text { no reported } \\
\text { comparison }\end{array}$ & $\begin{array}{l}\text { not } \\
\text { consistent }\end{array}$ & $\begin{array}{l}\text { relatively } \\
\text { good }\end{array}$ \\
\hline $\begin{array}{l}\text { Computer } \\
\text { resoirces }\end{array}$ & insignificant & moderate & moderate & significant \\
\hline
\end{tabular}

is then specified for a particular device structure and bias condition, and various numerical methods and algorithms are used for the solution of the electronic wave functions. The boundary conditions of the Schrödinger equation and the normalization of the wave functions are critical for obtaining the correct solutions. The boundary conditions used in the envelope function model and other quantum device models will be listed in Table 1 and discussed briefly when RTD quantum models are summarized.

In the envelope function model, electronic conduction in quantum systems is represented by the transmission coefficient based on the Landauer and Büttiker [15], [16] formulation. The transmission $T\left(E_{x}\right)$, as a function of the longitudinal electron energy, is the probability ratio of the incident and transmitted waves of a particular electron state, which is equivalent to the ratio of incident and reflected electron flux if the ratio of group velocities is also included.

To model the I-V characteristic of the RTD, a spectrum of electron eigenstates are taken to be incident from the contacts, and the electron waves are then subject to elastic and inelastic scattering by various scattering potentials included in the formulation of $V(\mathbf{r})$. The electron eigenstates in the contacts are assumed to be plane waves, and the reflected and transmitted wave functions are solutions of the Schrödinger equation for the specified RTD potential profile, including the scattering potential terms and the effective masses. Once the wave functions are found, the device current density can be computed

$$
J_{\text {tot }}=-q \hbar \sum_{k} W(k) \operatorname{Im}\left[\psi_{k}^{*}(x) \frac{1}{m^{*}(x)} \frac{\partial \psi_{k}(x)}{\partial x}\right] \Delta k
$$

where a superposition over suitably normalized quantum states $\psi_{k}$ is performed. Equivalently, the current density can be evaluated using the Tsu-Esaki formula [17], and the total current density is obtained by summing the current density of each state over occupied states multiplied by their transmission probability

$$
\begin{aligned}
J_{\text {tot }}= & \frac{q m^{*} k_{B} T}{2 \pi^{2} \hbar^{3}} \int_{E_{C}}^{\infty} T\left(E_{x}\right) \\
& \cdot \log \left[\frac{1+\exp \left(\frac{E_{F}-E_{x}}{k_{B} T}\right)}{1+\exp \left(\frac{E_{F}-E_{x}-q V_{A}}{k_{B} T}\right)}\right] d E_{x}
\end{aligned}
$$

where $T\left(E_{x}\right)$ can be calculated from the wave functions available in the solution of the Schrödinger equation and the supply function obtained by integrating over $k_{\|}$has been incorporated. Other symbols in (5) are conventional.

More recent RTD models using envelope functions have incorporated additional important physical aspects such as space charge effects, the 2-D accumulation layer in the RTD emitter region, multiband effects, and phonon scattering. These aspects are discussed next.

Space charge effects occur in RTD's because of the impurity doping profile and the mobile electronic charge that exists in the quantum well(s) as well as in the emitter notch or accumulation region. Since the charge density responds to the same electrostatic potential it generates, one needs to solve for the self-consistent potential and charge distributions. This is usually carried out by solving Poisson's equation and the effective mass Schrödinger equation iteratively, followed by the current density calculation. Inclusion of self-consistency significantly modifies the device characteristics due to effects such as band bending, changes in the effective barrier height, the effective voltage drop across the barrier, and the shifting of the resonant state energies in the quantum well. Several versions of the self-consistent solution, with varying degrees of approximation, now exist. Ohnishi et al. assume in their calculation that thermal equilibrium is maintained outside the barriers while the injected electrons are transported ballistically through and between the barriers [18]. Cahay et al., however, assume quantum ballistic transport through the whole region between the contacts [19]. An important choice in such methods resides in which locations the injected carriers are assumed to be maintained in thermal equilibrium, since it plays a critical role in the quantitative results for the quantum well charge and the current density. Which assumption is more appropriate may depend on the specific device structure being modeled and the operating conditions. More details on self-consistent calculations can be found in the original work or in [20] and references therein.

One of the consequences of the self-consistent potential is the formation of the 2-D accumulation layer in the RTD emitter region due to the band bending, in which the injected electrons can scatter into the quantized states and then tunnel through the resonant energy levels with or 
without further scattering. This corresponds to process 2 in Fig. 1. Current transport through the emitter accumulation layer is important since it can change the device current significantly. Within the envelope function model, Fiig et al. [21] have addressed this problem using an additional term in the emitter charge expression to account for the quantized states in the emitter accumulation layer, so that a quantum repulsion effect is observed and the peak current evaluation includes the contribution from carrier transport through these states. A better treatment of the emitter accumulation layer is now incorporated in the kinetic model using Green's functions. For details, see references to the Green's function model for RTD's in Section III-B.

Multiband effects are important in RTD modeling because in the envelope function description, the periodic part of the true wave function is ignored or assumed to be the same for different materials. In addition, if the interaction of electrons in different bands is important for the evaluation of the RTD current, a single-band effectivemass equation is not adequate. The most obvious problem with single-band models is that they do not include band nonparabolicity, which is due to the well-known $\mathbf{k} \cdot \mathbf{p}$ repulsion of the light hole and conduction bands. The energy scale of the nonparabolicity effect is set by the bandgap and the energy difference between these two bands. Nonparabolicity in the conduction band implies that the effective mass increases as energy increases above the band minimum. This results in lower resonance energies than would otherwise be expected. The effect is especially important for RTD's using small bandgap semiconductors such as the InGaAs in the very important InGaAs/AlAs double-barrier structure, which is lattice-matched to InP. As a result of these effects, the single-band models usually predict the second turn-on of the RTD I-V characteristic at a much higher applied bias voltage than experimental data obtained in many RTD structures.

A second effect is more subtle but actually just as, or more, important. It is that the scale of the decay of the wave function in the barriers is also affected by the nonparabolicity. The simplest model that goes beyond the single-band model is Kane's two-band model, derived from the k.p theory [22]. Instead of the parabolic imaginary $E=$ $E_{g}-\hbar^{2} \kappa^{2} /\left(2 m^{*}\right)$ relation within the bandgap of the barrier semiconductor, where $\kappa$ is the magnitude of the imaginary wave vector, the energy dispersion can be approximated as $E=E_{g} / 2+\sqrt{E_{g}^{2} / 4-E_{g} \kappa^{2} \hbar^{2} /\left(2 m^{*}\right)}$. Examination of this equation shows that this "imaginary" band links the light hole and conduction-band extrema. Thus, at a given energy in the bandgap, $\kappa$ is smaller than it would be in the single-band model. Since the decay lengths are just $1 / \kappa$, they are significantly longer in the two-band model. Transmission, and thus currents, is exponentially dependent on the decay length. This can easily result in the single-band model's underestimating the current density by a factor of two or more for typical devices of interest. Furthermore, the single-band model is useless for the interband tunneling devices. For example, in the InAs/AlSb/GaSb RITD shown

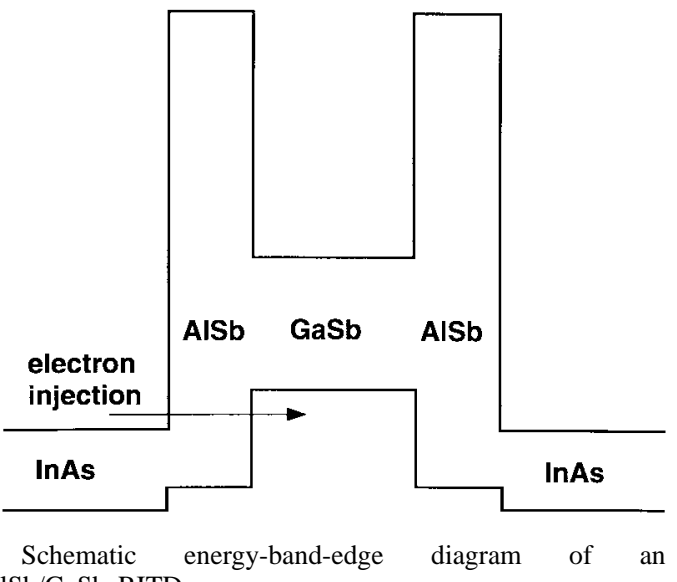

Fig. 3. Schematic ent
InAs/AlSb/GaSb RITD.

in Fig. 3, the electron is incoming in the InAs conduction band. The band alignment is such that the valence band of $\mathrm{GaSb}$ lies above the conduction band of InAs. Thus, that same electronic wave function is hole-like in the $\mathrm{GaSb}$ region. The two-band model is the simplest framework for incorporating this kind of situation, as it includes one conduction band and one valence (light hole) band in each material.

To incorporate multiband effects, Schulman introduced the two-band model into RTD modeling [23]; Rousseau et al. incorporated the multiband tight-binding model for RTD's [24], which was then further improved by several authors. For example, the ten-band nearest neighbor $s p^{3} s^{*}$ model [25] has been extended very recently to give improved material parameters and modeling results of RTD I-V characteristics by Boykin [26] and Bowen et al. [27].

Within the effective mass-envelope function framework, several formulations incorporating optical phonon scattering processes have been attempted with varying degrees of success. Chevoir et al. [28] treat the incoherent current transport as a sequential process from an occupied emitter state to a localized state in the well. Turley et al. performed calculations of resonant tunneling assisted by localized phonons in double-barrier structures [29]. Roblin et al. [30] employ a multiple sequential scattering algorithm to calculate scattering assisted tunneling currents. Cai et al. [31] use phonon annihilation and creation operators to form the Hamiltonian of the electron-phonon interaction, and this scheme was employed by $\mathrm{Fu}$ et al. [32] to calculate the phonon-assisted tunneling currents. In another approach, Stone et al. [33] and Zohta et al. [34] make use of the optical potential in analogy to its use in neutron scattering theory [35], in which an imaginary potential $-i W_{0}$ is introduced into the Hamiltonian to treat the scattering problem. Based on their work, Sun et al. [36] have improved the model to obtain results in good agreement with the experiment. At present, however, no single existing envelope function model can accurately predict the I-V characteristics for the whole range of RTD design parameters. A simple, elegant approach for the accurate design of $\mathrm{I}-\mathrm{V}$ characteristics of realistic RTD structures is desired but may not be feasible due to the fundamental 


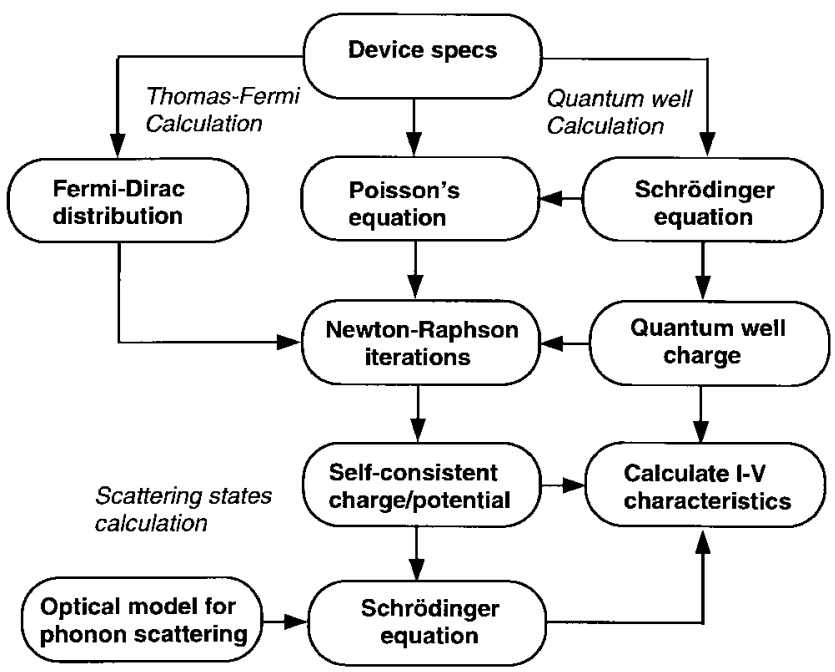

Fig. 4. An envelope function model for RTD's.

approximations used in this approach. Nonetheless, the envelope function model for RTD's and other quantum device structures is a valuable tool for simple device analysis in the quantum regime.

For an illustration of an approximate treatment of inelastic phonon scattering, a self-consistent RTD model including the optical potential for optical phonon scattering is described next in some detail. Its general procedure is outlined in Fig. 4.

The effect of the imaginary potential on electron motion can be seen directly from the time-dependent Schrödinger equation

$$
i \hbar \frac{\partial \psi}{\partial t}=\left[\frac{p^{2}}{2 m^{*}}+V-i W_{0}\right] \psi
$$

where $\psi=\psi(\mathbf{r}, t)$. Multiplying by $\psi^{*}$ on the left of (6) and taking the imaginary part, it follows that

$$
\nabla \cdot \mathbf{J}+\frac{\partial}{\partial t} \rho=-\frac{2 W_{0}}{\hbar} \rho
$$

where the electron density $\rho$ and current density $\mathbf{J}$ are defined, respectively, as

$$
\begin{aligned}
& \rho=\psi^{*} \psi \\
& \mathbf{J}=-\frac{\hbar}{m^{*}} \operatorname{Im}\left(\psi^{*} \nabla \psi\right) .
\end{aligned}
$$

It is seen from (7) that in the steady state, the electron current density is attenuated by the optical potential at a rate of $2 W_{0} / \hbar$, which corresponds to a scattering time $\tau=$ $\hbar / 2 W_{0}$. The simple form of the imaginary potential implies that the one-electron motion decays exponentially with time. On the other hand, one can see that the probability of finding electrons in the quantum well $|\psi|^{2}$ is reduced corresponding to an electron mean free path given by $\lambda=v \tau$, where $v=\sqrt{2 E / m^{*}}$ is the electron velocity for an electron state and $E$ is the longitudinal electron energy. We have dropped the subscript $x$. A damping constant $\gamma$ for the wave function in the quantum well can then be defined accordingly

$$
\gamma=\frac{1}{2 v \tau}=\frac{1}{\tau} \sqrt{\frac{m^{*}}{2 E}} .
$$

This allows us to express the electron wave function in the well as a linear combination of damping waves $e^{i(k+i \gamma) x}$ and $e^{-i(k+i \gamma) x}$ for the solution of the Schrödinger equation with the imaginary potential. This form for the wave function exhibits clearly that current conservation for the electronic state is not preserved. This is fundamentally due to the lack of unitarity of the Hamiltonian, which includes the complex potential.

When applied to the phonon-assisted tunneling calculation for RTD's in the growth direction $x$, the timeindependent Schrödinger equation now becomes

$$
\begin{aligned}
\frac{-\hbar^{2}}{2} \frac{\partial}{\partial x} & {\left[\frac{1}{m^{*}(x)} \frac{\partial \psi(x)}{\partial x}\right] } \\
- & {\left[E-V(x)+i W_{0}(E, x)\right] \psi(x)=0 }
\end{aligned}
$$

where $m^{*}$ is now a variable electron effective mass, depending on the material composition of the individual layers, and $1 / \tau$ is the scattering rate of a hot electron in the quantum well that is derived using Fermi's golden rule. The value of $1 / \tau$ can be approximated using the bulk scattering rate for optical phonons.

For simplicity, and as a good approximation for hot electrons, the bulk scattering rate for optical phonon scattering in the specific material is used similarly to typical Monte Carlo simulations

$$
\begin{aligned}
& \frac{1}{\tau}=S_{\mathrm{op}}\left[1+\frac{2}{\exp \left(\hbar \omega_{\mathrm{op}} / k_{B} T\right)-1}\right], \quad E-E_{R} \geq \hbar \omega_{\mathrm{op}} \\
& \frac{1}{\tau}=S_{\mathrm{op}}\left[\frac{1}{\exp \left(\hbar \omega_{\mathrm{op}} / k_{B} T\right)-1}\right], \quad\left|E-E_{R}\right|<\hbar \omega_{\mathrm{op}}
\end{aligned}
$$

where $E_{R}$ is the resonant energy in the quantum well and $S_{\mathrm{op}}$ is a prefactor of the scattering rates, adjustable to fit the experimental results. $\hbar \omega_{\mathrm{op}}$ is the energy of the optical phonon, $k_{B}$ is the Boltzmann constant, and $T$ is the absolute temperature. If we designate $N_{B}$ to be the phonon population, or the Bose-Einstein factor in these equations, then (12) can be read as $S_{\mathrm{op}}\left[\left(N_{B}+1\right)+N_{B}\right]$, representing the case where both emission and absorption of the optical phonon take place, whereas (13) becomes $S_{\mathrm{op}} N_{B}$, which is for the absorption case alone. The inelastic scattering potential is therefore energy dependent and related to the material used for the quantum well region in the RTD and to temperature.

In this approach, the total transmission $T_{\text {tot }}$ is taken to be the sum of the coherent transmission $T_{\mathrm{coh}}$ and incoherent transmission $T_{\text {incoh. }}$. An attenuation coefficient is defined as $A=1-T_{\mathrm{coh}}-R_{\mathrm{coh}}$, where $R_{\mathrm{coh}}$ is the coherent reflection coefficient. After a single collision event, the attenuated part is assumed to tunnel in both the $-x$ and $+x$ directions in proportion to the transmission coefficient for each barrier, denoted respectively as $T_{R}$ for the right (the second) barrier 
RTD with Phonon Scattering

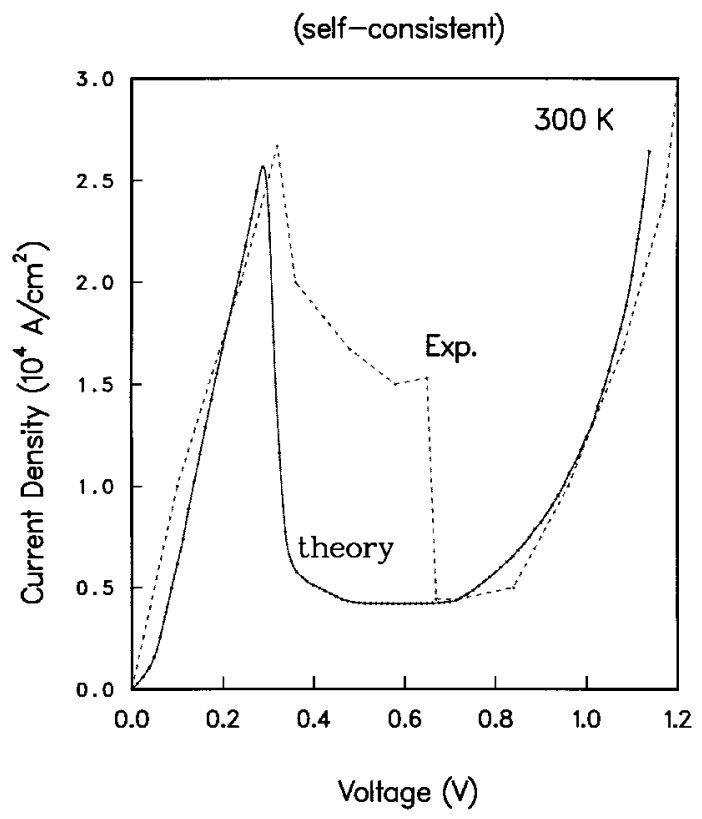

Fig. 5. Modeled and measured RTD I-V characteristics using the envelope function model with parameter adjustment.

and $T_{L}$ for the left (the first) barrier. Approximate analytical expressions have also been derived for these coefficients. To evaluate the transmission from region $\mu$ to region $\nu$, we use the squared ratio of the transmitted electron flux to the incident flux

$$
T_{\mu \nu}=\frac{\left|C_{\nu}\right|^{2}}{\left|A_{\mu}\right|^{2}} \frac{k_{\nu}}{k_{\mu}} \frac{m_{\mu}^{*}}{m_{\nu}^{*}}
$$

where $C_{\nu}$ is the transmitted wave amplitude, with associated effective mass $m_{\nu}^{*}$ and wave vector $k_{\nu}$, while $A_{\mu}$ is the incident wave amplitude, with $m_{\mu}^{*}$ and $k_{\mu}$ in the respective regions. The wave amplitudes are available from the numerical solution to (11) for a particular selfconsistent potential $V(x)$ obtained using the procedure outlined previously. The total transmission through the double-barrier structure is then

$$
\begin{aligned}
T_{\mathrm{tot}} & =T_{\mathrm{coh}}+T_{\text {incoh }} \\
& =T_{\mathrm{coh}}+\frac{T_{R}}{T_{L}+T_{R}}\left(1-T_{\mathrm{coh}}-R_{\mathrm{coh}}\right) .
\end{aligned}
$$

The total current density is evaluated according to (5) using the total transmission $T_{\text {tot }}$.

A comparison between calculated and measured RTD $\mathrm{I}-\mathrm{V}$ characteristics is displayed in Fig. 5. Note, however, that good agreement between theory and experiment such as this often involves parameter adjustment for the scattering rate $1 / \tau$. Applying this approach to RTD's with varying design parameters is not as successful in obtaining good agreement between modeled and measured results. In general, accurate prediction of RTD I-V characteristics cannot be claimed. Moreover, as will be discussed next, there are fundamental limitations to the coherent model, especially when a unified treatment of the electron and its interactions with the surrounding microparticles is desired.

\section{B. Kinetic RTD Models}

The envelope function models are classified as dynamic models because they model the time-dependent or dynamic device behavior based on the equation of motion of the system, i.e., the time-dependent Schrödinger equation. However, this framework cannot in principle treat the timeevolution of a system with internal force terms such as inelastic phonon scattering. For internal kinetics to be included, one has to invoke a kinetic equation such as the Boltzmann transport equation, in which internal forces are explicitly or implicitly incorporated. The pursuit of quantum kinetic models of RTD's was first motivated by the desire for a correct treatment of the inelastic electronphonon scattering process. The density matrix and Wigner function models were then developed with this goal in mind. A great deal of work has been performed on the application of Wigner functions to RTD modeling [37]-[40], and progress has generated very valuable information about RTD device properties. Although models based on the Wigner function and the density matrix are based on established principles, however, phonon scattering in these models is treated semiclassically within the relaxation time approximation. The present authors' experience is that consistent results for RTD I-V characteristics using the Wigner function approach have not always been obtainable over a range of device parameters when compared with experiment [41]. Very recently, the Green's function model has shown further improvement on the modeling of phonon scattering and other electron interactions and has generated encouraging results. It is currently under active development.

In this section, we describe and compare features of kinetic models. Some degree of mathematical formalism is unavoidable in discussing this topic, but an attempt was made to make this section self-contained, and it may be skipped without sacrificing continuity.

In principle, the fundamental difference between the coherent (or dynamic) and kinetic models is that the former is based on the single-particle picture using the pure state description, while the latter is based on the many-particle picture with mixed state description. Note, however, that in practice, a kinetic RTD model can be reduced to the singleparticle picture with the mixed state description retained, such as in the one-particle density matrix formulation. Because of this fundamental difference, the coherent models cannot treat interactions between the microparticles on a rigorous basis. This difference can also be traced to the nature of the model function. In coherent models, the envelope wave function is a one-particle, pure state wave function assumed to have phase coherence over the entire device, an interacting many-particle system. The phase coherence is clearly not preserved for electrons experiencing inelastic phonon scattering. For the same reason, the envelope function is not capable of properly modeling electron interactions in environments such as in the device contacts, where energy and particle exchanges take place. This is consistent with the central equation of the coherent 


\section{Phonon Scattering in RTD}

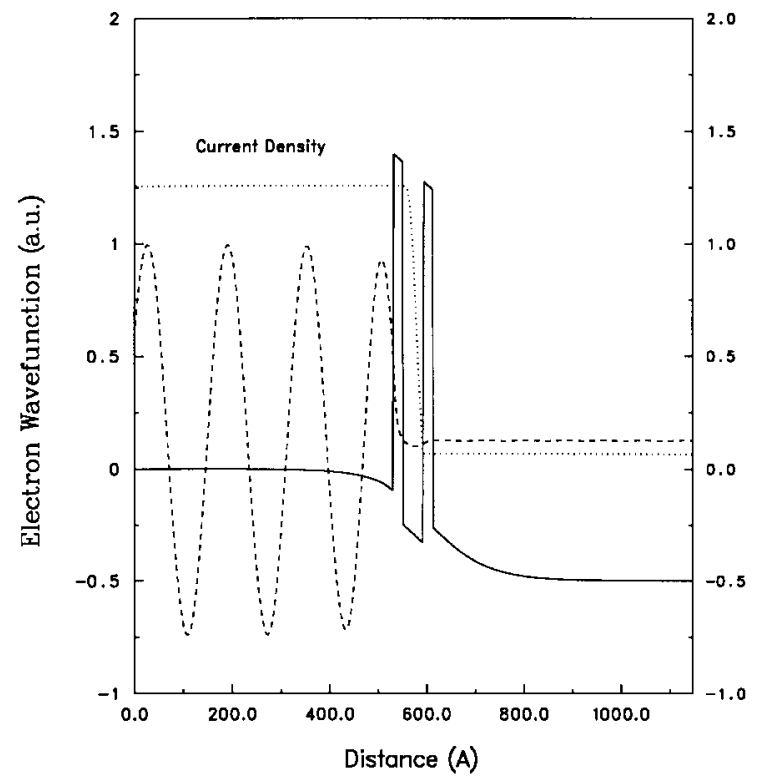

Fig. 6. Electron current and wave function due to phonon scattering using the optical potential approach.

model. In the time-reversible effective mass Schrödinger equation for an electron state, the state does not change its eigenenergy during its time evolution. Accordingly, this is a pure state description, which cannot treat electron-phonon and electron-electron interactions if they are strong enough such that the state energy changes and its phase coherence is destroyed. This results in a mixed state. In the optical model of optical phonon scattering within the envelope function framework, the energy, particle, and current conservation principles do not hold, since there is no correlation between the electron states being computed. This is deducible from Fig. 6, in that the electron wave function and the current are greatly attenuated, but there is no correlation to include the effect of this loss of current in one state to that of another in this framework.

When strong electron interactions must be included, one has to go beyond simple, single-particle, pure quantum states. Due to the statistical nature of quantum theory, a definite, conserved Hamiltonian for the Schrödinger equation for the system cannot be specified, and the electron can be described in terms of a mixed state. In kinetic quantum transport models, a quantum device is considered to be a particle ensemble, characterized by the density matrix, the Wigner (distribution) function, or Green's functions. As a rigorous understanding of these model functions involves advanced quantum physics concepts and extensive formulation, we give here only a brief and superficial discussion to introduce the conceptual differences with the coherent model and to identify the model functions and the central equations of these approaches.

First we separate the problem into two parts, i.e., the device under consideration and the environment outside the device. Then, let $x$ describe the device system in which we are interested and let $y$ describe the part of the universe outside the system. The density matrix is defined as

$$
\rho\left(x, x^{\prime} ; t\right)=\int \psi(x, y ; t) \psi^{*}\left(x^{\prime}, y ; t\right) d y .
$$

Its temporal evolution is given by the Liouville equation

$$
i \hbar \frac{\partial \rho}{\partial t}=[H, \rho]+\mathcal{L} \rho
$$

where [ ] is the Poisson bracket and $\mathcal{L}$ is the Liouville operator. Equation (18) can be written in the following form for device modeling

$$
\begin{aligned}
\frac{\partial}{\partial t} \rho\left(x, x^{\prime} ; t\right)= & \frac{i \hbar}{2 m^{*}}\left[\frac{\partial^{2}}{\partial x^{2}}-\frac{\partial^{2}}{\partial x^{\prime 2}}\right] \rho\left(x, x^{\prime} ; t\right) \\
& -\frac{i}{\hbar}\left[V(x, t)-V\left(x^{\prime}, t\right)\right] \rho\left(x, x^{\prime} ; t\right) .
\end{aligned}
$$

Based on Wigner's definition, the Wigner function is the Fourier transform of the one-particle density (matrix) operator

$f(x, k, t)=\frac{1}{\pi} \int_{-\infty}^{\infty} d y \psi^{*}(x+y ; t) \psi(x-y ; t) e^{2 i k y}$.

Alternatively, by performing the Wigner transformation for the Schrödinger equation, one arrives at the Liouville transport equation, which gives the time evolution of the Wigner function for device modeling

$$
\begin{aligned}
\frac{\partial f(x, k)}{\partial t} & \\
= & \frac{-\hbar k}{m^{*}} \frac{\partial f(x, k)}{\partial x}+\left[\frac{\partial f(x, k)}{\partial t}\right]_{C}-\frac{1}{2 \pi \hbar} \int_{-\infty}^{\infty} d k^{\prime} \\
& \left\{2 \int_{0}^{\infty} d y \sin \left(\left[k-k^{\prime}\right] y\right)\right. \\
& \left.\cdot\left[V\left(x+\frac{y}{2}\right)-V\left(x-\frac{y}{2}\right)\right]\right\} f\left(x, k^{\prime}\right) \\
= & \frac{-\hbar k}{m^{*}} \frac{\partial f}{\partial x}+\sum_{\lambda_{\text {odd }}} \frac{1}{\lambda !} \frac{\partial^{\lambda} V(x)}{\partial x^{\lambda}}\left(\frac{\hbar}{2 i}\right)^{\lambda-1} \frac{\partial^{\lambda} f}{\partial p^{\lambda}} \\
& +\left[\frac{\partial f(x, k)}{\partial t}\right]_{C}
\end{aligned}
$$

where the time derivative with subscript $C$ denotes a term due to scattering and $V$ is the potential. In a simple form, the device is assumed to obey the Liouville equation

$$
\frac{\partial f}{\partial t}=\frac{\mathcal{L}}{i \hbar} f+\mathcal{C} f
$$

where $\mathcal{C}$ is the collision operator

$$
\mathcal{C} f(x, k, t)=\int d k^{\prime}\left[W_{k k^{\prime}} f\left(x, k^{\prime}, t\right)-W_{k^{\prime} k} f(x, k, t)\right]
$$

and $W_{k k^{\prime}}$ is the transition rate from $k^{\prime}$ to $k$. Equation (22) expresses the time evolution of the Wigner function in terms of the Liouville operator, representing the ballistic motion, and a collision operator to include dissipative interactions such as the optical phonon scattering. The 


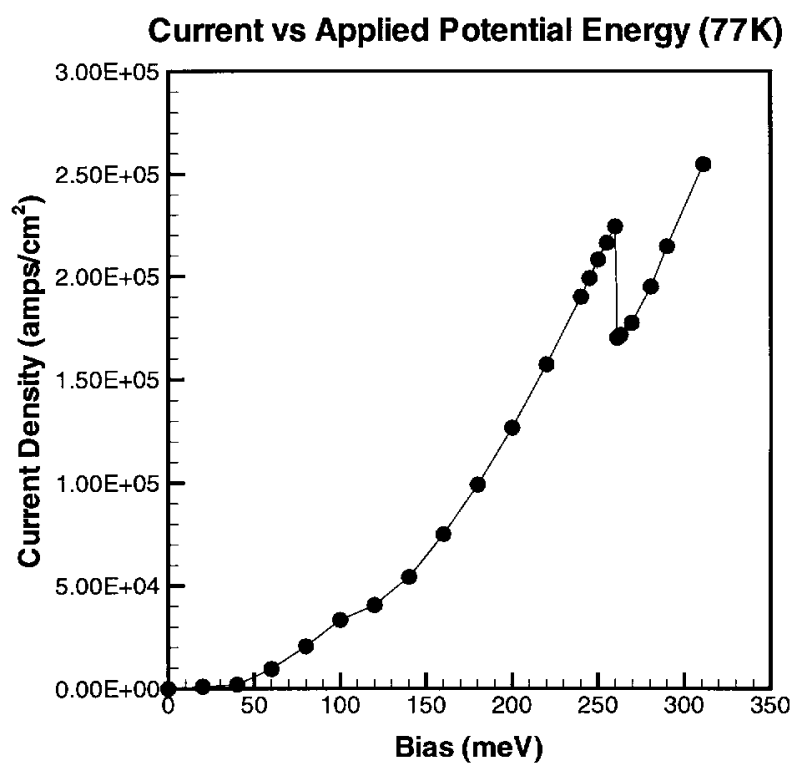

Fig. 7. Current density versus voltage for an RTD structure using the density matrix model. Reproduced from [42] with permission.

resultant quantum Liouville equation may be considered as the quantum Boltzmann transport equation. In fact, in the classical limit, (21) is reduced to the semi-classical Boltzmann equation

$$
\begin{aligned}
\frac{\partial f}{\partial t} & =\frac{-\hbar k}{m^{*}} \frac{\partial f}{\partial x}+\frac{\partial v}{\partial x} \frac{\partial f}{\partial p}+\left(\frac{\partial f}{\partial t}\right)_{C} \\
& =-v \frac{\partial f}{\partial x}-F \frac{\partial f}{\partial p}+\left(\frac{\partial f}{\partial t}\right)_{C}
\end{aligned}
$$

where $f$ is the familiar Boltzmann distribution function. Conceptually, since the transport equation is written in terms of a sum of many state functions, it is capable of describing mixed quantum states; the mixing of states occurs due to the scattering terms in (21). This kinetic model offers a proper conceptual basis for dealing with quantum transport phenomena and has generated many useful insights into RTD physics and device properties. Fig. 7 shows an I-V curve modeled using the density matrix approach for a 200-nm RTD structure, with two 5-nm, 300-meV barriers separated by a 5-nm well at 77 $\mathrm{K}$. More details of this work can be found in [42]. In Fig. 8, a comparison between RTD I-V curves modeled by the (coherent) tunneling theory and by the Wigner function model is shown for a GaAs/AlGaAs RTD structure consisting of a 4.5-nm quantum well bounded by 2.8 $\mathrm{nm}$ barriers. The scattering effects on the peak and valley current densities in the Wigner function model are evident. For more details of comparison between these modeling results, the reader is referred to the original paper [12]. The major accomplishments, shortcomings, and detailed formulations of the density matrix and Wigner function approaches for RTD's have been summarized in recent reviews [42], [43].

RTD models based on nonequilibrium Green's functions have gained increasing interest recently because this approach offers a comprehensive physical basis for the

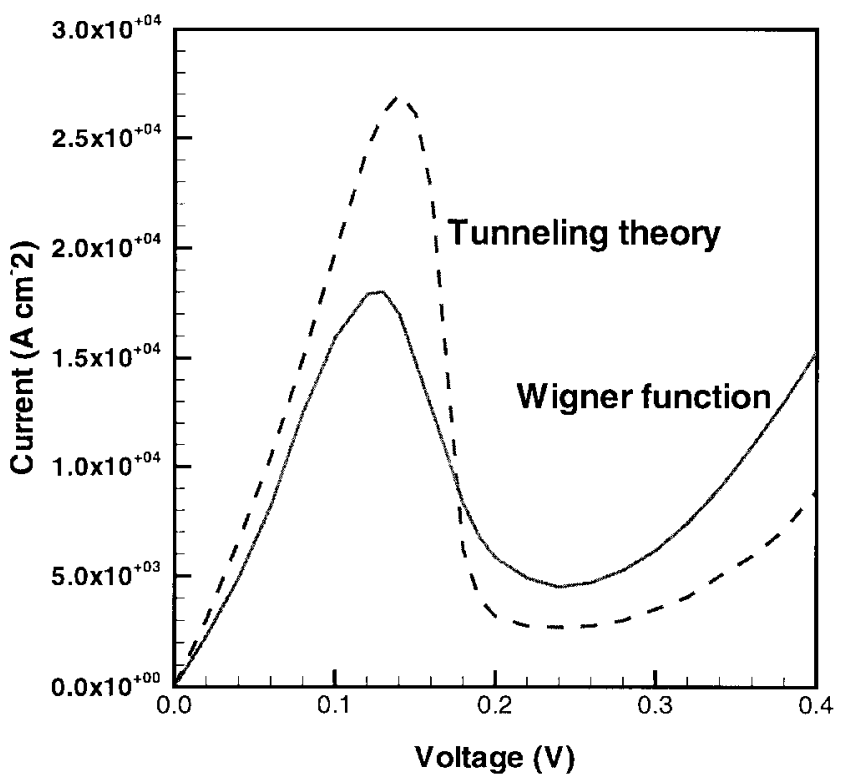

Fig. 8. Current density as a function of voltage for a model RTD using the Wigner function model. Reproduced from [12] with permission.

treatment of interactions between microparticles in the device system [44], [45]. Electron-electron interactions in the contact regions, including the emitter accumulation layer, electron-phonon interactions, and other scattering processes, can also be readily incorporated in a unified formulation. This approach has produced improved results and more microscopic information in the device over the other RTD models [46].

Green's functions are utilized to treat many physical phenomena. For example, in electromagnetic theory, they are used to calculate the response of a system to external, point excitations. More specifically, Green's function determines the response in $\mathbf{r}$ at time $t$ due to an excitation in $\mathbf{r}^{\prime}$ at time $t^{\prime}$. For transport problems such as in RTD modeling, one takes the RTD current to be the response to excitations. Nonequilibrium Green's functions can be used, typically in matrix form, as a computational vehicle that contains the information about device response to various excitations, such as applied voltage. Moreover, various scattering sources, such as phonons, electrons, impurities, interface roughness, etc., can be taken as internal excitations, and the contributions to the current from the individual excitations can then be calculated by summing up all the responses.

Several different but related nonequilibrium Green's functions are required to encompass the increased microscopic information contained in the Green's function model for RTD's. The model functions consist of a number of different Green's functions, i.e., the retarded and advanced Green's functions $G^{R}, G^{A}$, the correlation function $G^{<}$, and the self-energies $\Sigma^{R}, \Sigma^{A}, \Sigma^{<}$. Heuristically, these functions may be considered, respectively, as the electron wave response at different locations with respect to the excitation, the correlation between different states at different times, and the Hamiltonians of the scattering 
potentials. As the formalism needed to define and describe the Green's functions correctly is nontrivial, we refer the reader to the description of Green's functions in quantum physics [47] and the treatment by Keldysh, Kadanoff, and Baym (the KKB formalism) [48], [49]. Datta has recently written an excellent book explaining the use of Green's functions for mesoscopic transport problems including RTD calculations [50].

The central equation for the Green's function approach can be considered to be Dyson's equation [51]. Starting from Dyson's equation in the Keldysh formalism, Datta and Lake formulated a set of equations for the Green's functions for RTD steady-state transport calculations, which yield dc I-V characteristics [45], [52]. For example, the equations for the retarded Green's function $G^{R}$ and the correlation function $G^{<}$are

$$
\begin{aligned}
& {\left[E-H_{0}(\mathbf{r})\right] G^{R}\left(\mathbf{r}, \mathbf{r}^{\prime} ; E\right)} \\
& -\int d \mathbf{r}_{1} \Sigma^{R}\left(\mathbf{r}, \mathbf{r}_{1}\right) G^{R}\left(\mathbf{r}_{1}, \mathbf{r}^{\prime} ; E\right)=\delta\left(\mathbf{r}-\mathbf{r}^{\prime}\right) \\
& G^{<}\left(\mathbf{r}, \mathbf{r}^{\prime} ; E\right)=\int d \mathbf{r}_{1} d \mathbf{r}_{2} G^{R}\left(\mathbf{r}, \mathbf{r}_{1} ; E\right) \\
& \times \Sigma^{<}\left(\mathbf{r}_{1}, \mathbf{r}_{2} ; E\right) G^{R^{*}}\left(\mathbf{r}^{\prime}, \mathbf{r}_{2} ; E\right) .
\end{aligned}
$$

Other equations related to the self-energies are also needed for the model but will not be listed here. Further approximations have also been used to simplify the formulation and numerical work.

RTD models based on nonequilibrium Green's functions can include the electron-microparticle interaction in a unified framework. This gives more insight and microscopic information about the current transport, the effects of inelastic scattering on the occupation of the energy levels, the density of states, the energy distribution of the current density, and the power density. These can all be calculated for practical device structures under high bias conditions, which is not possible with other existing RTD models. Recent efforts at Texas Instruments by the Nanoelectronics Group, in collaboration with others, have produced nanoelectronic modeling (NEMO) software utilizing nonequilibrium Green's functions to attempt stateof-the-art device modeling capabilities for RTD's and to provide a comprehensive quantum device modeling tool for nanoelectronic devices (see [46] by Klimeck et al.). This model includes the effects of quantum charging, band structure, incoherent scattering from alloy disorder, interface roughness, ionized dopants, and acoustic and polar optical phonons. The relevant publications have indicated that the NEMO program may be more accurate for modeling RTD I-V characteristics and other steady-state properties than previous RTD models. On the other hand, the development of KKB-based time-dependent modeling is still in its infancy. So far, only simplified transfer Hamiltonians have been used [53], [54]. It is worth noting, however, that RTD's are capable of operating in the terahertz frequency range, and time-dependent models may not be that important for the frequencies of interest in the near future for digital circuit applications being considered. In addition, as is the case for other quantum kinetic device modeling, significant computer resources are demanded, both for memory and CPU time. Nonetheless, RTD models based on nonequilibrium Green's functions represent a remarkable advance in quantum transport modeling, and further enhancement of their capabilities is expected.

In Table 1, the major features of various RTD models discussed above are summarized to give a sketchy outline of these models. Because we try to give a very simplified summary in a tabulated form, some terms in the table are loosely used. For example, there, the Green's functions really mean nonequilibrium Green's functions. The density matrix model is categorized to be kinetic only in principle; it is not strictly true, however, for the RTD models based on the one-particle density matrix formulation.

A brief discussion on the boundary conditions in RTD modeling is in order. As alluded to in the beginning of this section, the boundary conditions must reflect the open nature of the device boundaries. Mathematically, values and derivatives of the model functions at the boundaries may be formulated for the solution of the model equations. Listed in the table are the quantum transmitting boundary method (QTBM) proposed by Lent and Kirkner [55] and the injected distribution function. The QTBM approach has gained more applications recently. Generally speaking, the essence of this approach is to apply mixed boundary conditions by fixing the values of linear combinations of the model functions and their gradients at the device boundaries. The physical conditions at the boundaries and interfaces can then be enforced during the entire solution process. For a specific example of how it is implemented, see [56]. The injected distribution function approach is a traveling wave condition in that the boundaries emit a quasicontinuum of eigenstate electrons with their wave vectors distributed according to the thermal equilibrium distribution. The integral of these eigenstate electrons yields the doping concentration at the contacts. One can appreciate that this approach reflects the open nature of the device contacts and offers a proper formulation for the normalization of the model functions. Similar treatment of boundary conditions is also applicable in RTD models based on the envelope function approach.

In addition, we may note the difference between the nonequilibrium Green's function model and the other kinetic RTD models. In the entry of phonon scattering, under the density matrix and Wigner function models, the relaxation time approximation implemented in the collision term in these RTD models may also be characterized as being Markovian. The Markov approximation assumes that the evolution of the device system (through phonon scattering in this context) does not depend upon its past history. This is reflected in the density matrix formulations, where $\rho\left(x, x^{\prime}, t\right)$ is a one-time model function. This assumption, however, is not made in the nonequilibrium Green's function model. Therefore, the Green's function model has an additional degree of freedom capable of modeling nonMarkovian processes in the device. Further study of this point can be extended in relationships between the density 
matrix, Wigner function, and Green's function formalisms. Treatment of other scattering processes included in the envelope function and the Green's function model can, in principle, be implemented in the density matrix and Wigner function models, but no report has been published to our knowledge.

\section{Other RTD Models}

Based on the well-known Esaki-Tsu integral formula for the tunneling current, many modifications and improvements have been made, such as the work by Coon et al. [57] and Chang et al. [58]. Analytic models for the RTD current such as those offer simple formulas relating the most important physical ingredients of RTD operation but do not adequately predict experimental $\mathrm{I}-\mathrm{V}$ behavior. On the other hand, as RTD device and circuit developments advance to the stage of practical circuit innovation and implementation, there is a need to bridge the gap between the available RTD physical models and circuit simulation. The RTD quantum models are too complicated and generally not accurate enough to be directly called from a circuit simulator. Recent attempts have begun to incorporate the RTD NDR into circuit simulation models based on SPICE-type computeraided design tools [59]. Therefore, simple analytic models that closely relate both the essential RTD physical parameters and measured $\mathrm{I}-\mathrm{V}$ characteristics are desired. Work in this area follows analytical approximations for the I-V characteristics based on transmission calculations with adjustable empirical parameters linked as closely as possible to real physical quantities. It has been shown that such models can accurately reproduce experimental I-V characteristics [60], which should be a very valuable tool for realistic RTD device and circuit development.

There are several important issues to note when comparing theoretical and experimental results, including the uncertainties in the material and doping profiles of the device, the possible error in the growth data, the material parameters employed such as effective masses and band offsets, and the effects of imperfectly known parasitic impedances on the measured I-V curve. These factors make it very difficult to obtain an accurate prediction or reproduction of experimental $\mathrm{I}-\mathrm{V}$ characteristics and demand more accurate characterization and calculation of material and structural parameters pertinent to RTD modeling calculations.

Last, there is a well-known issue that is conceptually simple but difficult to include in practice: the inclusion of the series and contact resistances. These resistances modify the device I-V characteristics. It is difficult for all the above models to include an accurate evaluation of these resistances. Usually, a rough estimate or measurement is made to fit the measured I-V curve, which may not be satisfactory for circuit designers if the RTD's being used have a large fluctuation in their values for the resistance, and therefore in their peak and valley voltage values. A recently proposed technique to determine the RTD I-V characteristics experimentally based on microwave reflection coefficient measurement may be useful in this regard
[61]. The importance of this issue will become clear in the next section, where RTD I-V parameters for logic circuits are discussed.

\section{DEVICE PRoperties}

We now examine RTD dc properties, emphasizing only those that affect potential digital logic circuit applications, since this is where the major impact is expected. Before specific RTD dc properties required by its logic applications are discussed, we note that there are also serious considerations for analog applications, signal processing, and A/D converter applications of RTD's, which are not covered in this paper.

The most important property of an RTD lies in its NDR, which is sustainable to very high frequencies and offers very fast switching speed. Of course, the time-dependent device properties possess interesting and subtle effects that have absorbed a great deal of effort, particularly in relation to device transients and high-frequency behavior. Recent results have shown that RTD's can produce small amounts of power at frequencies above $800 \mathrm{GHz}$, and calculations predict an NDR response in the terahertz range.

An analysis of RTD switching speed requires solving the central transport equations in the time domain. The resulting current transient information can be Fourier transformed into the frequency domain to obtain the admittance, which can in turn be used to construct a small signal circuit model for the analysis of the high-frequency properties. RTD switching characteristics have been investigated by many authors. Liu and Sollner have recently written a comprehensive review covering many aspects of RTD highfrequency behavior (see [62] and references therein). Despite the impressive high-frequency oscillations that have been demonstrated [63] and theoretical predictions that terahertz frequencies are obtainable, current devices produce only very low levels of output power and have limited application as power sources. It is hoped that recently proposed schemes for coherently combining the power of RTD's in array form can overcome this limitation. We will not discuss high-frequency aspects of RTD's in this paper.

For realistic applications of RTD's in digital circuits, some of the questions that need to be addressed are: What is required of the dc $\mathrm{I}-\mathrm{V}$ characteristic for typical digital circuits, e.g., logic gates? How likely is it that these requirements can be attained? What are the major issues concerning the choice of material system and structural considerations? In addition, there exist other quantum devices, notably the resonant tunneling transistor (RTT), the resonant tunneling hot-electron transistor (RHET), the RITD, and the Esaki TD, all of which exhibit NDR characteristics. Is the RTD more attractive than the others? We will provide a brief comparison among some of those devices in terms of their main features and properties. Before we make the comparison, it is helpful to see what is required from the device NDR characteristics for digital logic applications.

First of all, it is important to note that two-terminal device logic circuits, i.e., using RTD's alone, have several limita- 


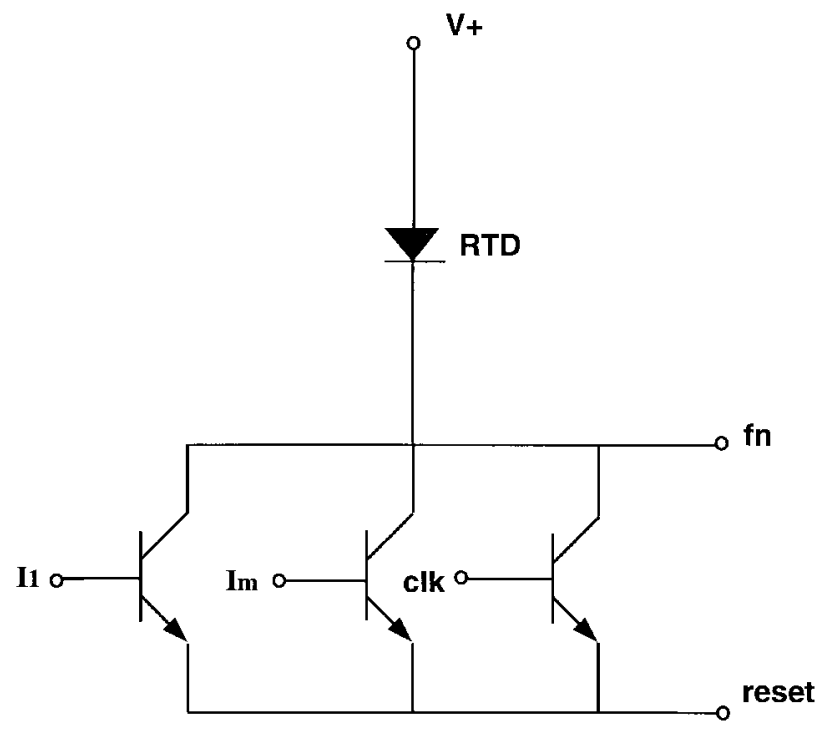

Fig. 9. An example of logic gate consisting of RTD's and HBT's.

tions and are not adequate for most circuit applications. That is the main reason that circuits employing conventional Esaki TD's did not materialize in the past. Recent advances in very-high-frequency transistors such as HBT's and HEMT's, however, and the possibility of integrating NDR devices such as RTD's with them, have changed the picture significantly. The combination of these devices now holds great promise for very high speed/functionality circuits. In the next section, we will mainly consider an RTD-HBT-based circuit as an illustration [58], but certainly RTD-HEMT based circuits and other circuits are also very important and are being pursued.

\section{A. NDR Characteristics and Parameters}

For illustrating the issue of the integration of RTD's and transistors, we take a typical RTD-HBT threshold logic gate as an example. Fig. 9 shows the logic gate configuration with $m+1$ HBT's as the logic driver and an RTD used as the load. This is referred to as a minority gate and can be employed to achieve many digital functions. The logic gate operates as follows. The input currents $I_{1}$ through $I_{m}$ change to cause changes in the current flowing through the RTD load. When the clock (clk) signal goes high, it will cause the total current through the RTD to increase. If more than $n$ inputs are high, the current through the RTD exceeds the peak current, causing a jump, and thus switches the logic state to the second positive differential resistance (PDR) region. This results in the $f_{n}$ node's going low. If less than $n$ inputs are high, the current through the RTD does not exceed the peak current and $f_{n}$ remains high. When the clock signal goes low, the previous output is maintained. When the reset line goes high, it will force all the transistors into cutoff. The current flowing through the RTD will fall below the valley current, and the $f_{n}$ node is pulled high. When the reset line goes back to zero, the $f_{n}$ node remains high. The logic operation can also be seen from Fig. 10, which shows the intersections of the RTD's I-V curve with the HBT's I-V

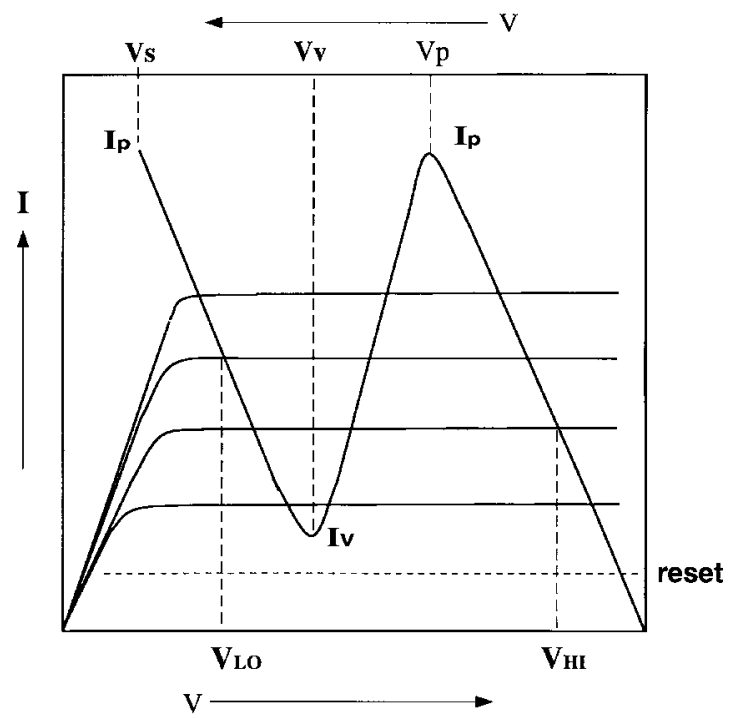

Fig. 10. Intersecting characteristics of RTD's and HBT's in a logic gate.

characteristic. The I-V curve of the basic RTD device can be divided into three branches and six interrelated NDR parameters as shown. There are two PDR branches, one before the peak point and one beyond the valley point, with the NDR region in between. Other important quantities are the peak current $\left(I_{P}\right)$, peak voltage $\left(V_{P}\right)$, valley current $\left(I_{V}\right)$, valley voltage $\left(V_{V}\right)$, and the peakto-valley current ratio (PVR). A voltage swing $\left(V_{S}\right)$ may be defined as the voltage at which the current reaches $I_{P}$ on the second PDR branch. Desired NDR characteristics can be analyzed in terms of the above parameters. The requirements on the RTD parameters can be considered relative to the noise margin, speed, and power dissipation for the logic gate.

When the HBT's are switched on by high (HI) input signals, the current flowing through the load RTD will intersect its PDR branch. If the current reaches $I_{P}$, the operating point will be switched to the $V_{S}$ point, producing a low output (LO) voltage, thus achieving a logic state. Conversely, a LO input signal at the HBT's input will set the RTD to a voltage near $V_{\mathrm{DD}}$, the power supply of the gate. Since the noise margin of the logic gate is related to the difference $V_{\mathrm{HI}}-V_{\mathrm{LO}}$, we require $V_{S}-V_{P}$ and thus $V_{V}-V_{P}$ to be as close to $V_{\mathrm{DD}}$ as possible. This demands a low value for $V_{P}$ and high values for $V_{V}$ and $V_{S}$.

The $I_{P}$ value sets up the logic relationship of the gate since it determines the point at which the logic state changes. However, it also depends on specific circuit driver configurations and therefore cannot be considered to be a stringent requirement. Generally, too large a value of the peak current is certainly undesirable from the viewpoint of power dissipation for future large-scale circuit integration. In addition, it takes time to reach a large peak current point and then decrease to the valley point between the different states. This is undesirable for high speed. For the logic circuit shown in Fig. 9, a value for $I_{P}$ of a fraction of a milliampere will usually suffice. 
Evaluating the valley current, and the related PVR, has been a major challenge, with the associated goals of understanding current transport, improving RTD models, and achieving high PVR's. In general, a low valley current density is desired, as it is necessary for switching and is also advantageous for low power consumption when largescale RTD circuits are integrated. On the other hand, merely seeking a very high PVR may be misleading and should be clarified. The PVR must be sufficiently large to provide the logic function with a good noise margin, but it is not the sole figure of merit of an RTD. As can be seen in Fig. 10, if the PVR of the RTD is too low, a noise signal may trigger the operating point near $\left(I_{P}, V_{S}\right)$ to move down along the NDR branch of the $\mathrm{I}-\mathrm{V}$ curve, reaching the valley point and then subsequently switching to an incorrect or faulty state. As a rule of thumb, a PVR of five would generally be adequate; a value of ten is quite sufficient, and further pursuit of a much higher PVR may not be necessary for logic applications.

It should be noted that the above discussion is an example for illustration. Since specific requirements for RTD characteristics may vary depending on the particular circuit configuration of different logic families and on different digital applications, the considerations may or may not apply for different digital circuits. For example, for RTD's used for large-scale memory applications, the PVR will be required to be as high as possible to reduce the standby power consumption.

\section{B. Material and Structural Considerations}

The NDR characteristics considered in the preceding section as representative device properties are a direct consequence of the carrier transport, described in Sections II and III. They are closely related to the material system and specific device structure. A great deal of research has been devoted to exploring the various options for the realization of optimum RTD's.

It is obvious that for the sake of future large-scale integration, the most desirable material system would be silicon based, being compatible with current silicon IC technology. In contrast to the successful HBT performance achieved in $\mathrm{Si} / \mathrm{SiGe}$ heterosystems [64], however, attempts at building RTD's in the $\mathrm{Si} / \mathrm{SiGe}$ system with acceptable I-V characteristics have not been encouraging, with PVR's not much larger than one [65], [66]. The most important relevant parameter is the energy-band offset, particularly the offset in the conduction band, because in most cases electron transport is desired due to its lighter effective mass as compared with holes. Although various schemes based on the SiGe alloy band structures have been proposed and tested, the conduction-band offset between $\mathrm{Si}$ and SiGe alloys has been limited to a few of tenths of an electron volt. This is not enough to produce the necessary quantum confinement for a strong resonant state in the DBQW structure. Studies on hole resonant tunneling in SiGe systems have also been carried out [67], [68]. Tsu [69] has proposed silicon-based quantum wells with $\mathrm{Si} / \mathrm{SiO}_{2}$ strained layer superlattice barriers, but the structure has not been demonstrated. This is probably due to difficulties in the fabrication technology and strong electron scattering's obscuring the resonance. Another possibility is to grow materials with different bandgaps that are lattice matched to silicon, for example, SiGeC materials [70]. There have also been recent proposals for quantum MOS transistors incorporating RTD's, but a real breakthrough in this area is yet to come.

For RTD's based on III-V compound materials, the original traditional material system, GaAs(well)/AlGaAs(barrier), has been extended to GaAs/AlAs, InGaAs/AlAs, InGaAs/InAlAs, InAs/AlSb, and others. The work on RTD's built from these material systems is extensive. For a summary of the various RTD characteristics achieved in these systems, see [71] by Seabaugh and Lake. RTD's made from these systems can achieve a very high peak current density, a sufficient PVR, and a very high speed index (i.e., the ratio of the peak current to the device capacitance).

At this time, the GaAs/GaAlAs and InGaAs/AlAs (on InP substrates) systems are the strongest candidates for actual circuit insertion. The InGaAs/AlAs system is clearly superior in terms of its intrinsic properties, but GaAs/GaAlAs IC fabrication technology is significantly more mature in general. The focus now is on low power at moderately high frequencies (tens of gigahertz), as there are other more conventional high-frequency solutions available if power is not the primary objective. In this regard, a variation of the InGaAs/AlAs system has come to the fore. It contains a modified InGaAs well with extra In inserted, either in the form of a higher alloy concentration than called for by lattice matching or a small number of pure InAs central layers [72]. Either way, the extra In lowers the resonance energy in the well so that the peak voltage is lowered correspondingly. The tradeoff is that the peak current decreases as the resonance is lowered, so a compromise is required. The lower voltage is absolutely essential if low power is to be achieved. For that purpose, an even better system is the InAs/AlSb/GaSb RITD, or a variation thereof, which will be explained shortly.

While it may be straightforward in principle to control the peak current density by properly designing the DBQW structure, it is not easy to realize this in practice. The tunneling current density essentially has an exponential dependence on the barrier thickness, which varies typically by one monolayer either way from the nominal thickness aimed for in the epitaxial growth. The window for optimum condition in the growth is narrow and often unforgiving, with no warrant of precise repetition. Therefore, iterative adjustment is usually needed for a desired current density. Further, it is difficult to obtain the small $V_{P}$ values predicted by calculations for reasons related to device contact and series resistances. The PVR is a combined result of the factors relating to the valley current transport mechanisms, material system, structural design, and processing technology. The valley voltage naturally has material and structural dependences that can be considered, as in Sections III and IV. Last, the swing voltage $V_{S}$ is related to the valley shape 
and the turn-on in the second PDR branch, which is very much related to the material parameters. For example, since the interaction between the conduction and valence-band electrons becomes more significant when the electric field strength increases with applied voltage, an early turn-on and a small $V_{S}$ are expected for materials with a narrower bandgap. In addition, it is easy to see that a low barrier material leads to earlier turn-on and a smaller voltage swing.

The above discussion indicates a strong dependence of the NDR on the material and structure. An initial design may take these factors into consideration, but since factors affecting the resultant NDR and I-V characteristics are highly nonlinear, modeling calculations to aid quantitative design are desirable. Previous material growth data and RTD processing parameters, if available, should be very valuable for a realistic assessment of expected RTD I-V characteristics. Design and process iterations are often used to obtain a desired device NDR characteristic.

In addition to the basic DBQW structure, several other RTD structures have been investigated for interesting resonant tunneling physics, for possible performance improvement, and for a variety of potential applications. Notable examples include polytype InAs/AlSb/GaSb RITD's based on resonant interband tunneling mechanisms [73]-[75], the Schottky-collector RTD's [76], the double- and triple-well RTD's, which generate multiple NDR characteristics for multiple-valued logic circuits [77], [78], and, in yet another direction, RTD's of lower dimensions, so called 1-D and zero-dimensional (0-D) RTD's [79], [80].

The main idea of an RITD is to combine interband tunneling in Esaki TD's and resonant tunneling in quantum well structures. These combinations became possible due to various types of band lineups in polytype heterostructures. An example is shown in Fig. 3, where electrons tunnel from the conduction band (InAs contacts) through an interband barrier into light hole states in the valence band ( $\mathrm{GaSb}$ quantum well). The peak current is caused by the tunneling through a resonant state in the quantum well, as in the case of conventional RTD's. As the bias voltage passes resonance, however, the electrons will experience the bandgap not only of AlSb but also of GaSb. Therefore, in principle, such devices are expected to have very high PVR's, and the published work has shown promising results. It may have a dramatically lower peak voltage but still with the high peak currents typical of InGaAs/AlAs without the extra In. The low voltage is due to the Type II band alignment: the InAs emitter conduction-band edge proximity to the $\mathrm{GaSb}$ quantum well valence band. The positive mass of the conduction band relative to the negative valence-band mass implies that there can be electrons satisfying the resonance condition even at very small biases. Fabrication of transistors in this material system has been demonstrated, but it is at a very early stage.

In a somewhat different version of RITD's, heterostructure $\mathrm{p}-\mathrm{n}$ junction TD's have also been demonstrated [81]. For example, in an InGaAs/InAlGaAs/InGaAs tunnel diode, the wide-bandgap barrier layer is inserted in between the two narrow-bandgap contact layers. The tunneling current in such tunnel diodes is determined not only by the doping profile, as in a homojunction Esaki TD, but also by the barrier width and height, which can be controlled to tune the device I-V characteristics, thus adding a degree of freedom in device design.

For Schottky-collector RTD's, the ohmic-contacted collector of a conventional RTD is replaced with a direct Schottky contact to the space charge layer near the second barrier, thereby eliminating the contact series resistance on that side. This reduced series resistance leads to an increased maximum frequency of oscillation. A 64-element monolithic Schottky-collector RTD oscillator array oscillated at $650 \mathrm{GHz}$ has been reported [82].

In 1-D RTD's, the electrons are laterally confined in the $y$ or $z$ directions using the convention of Fig. 1. Another novel example is the variable-area RTD, in which the size of the lateral confinement can be altered [83]. In 0-D or quantum dot RTD's, electrons are confined laterally as well as vertically, but weakly coupled to electron reservoirs. Resonant tunneling has been observed in these lower dimensional RTD structures. These lower dimensional structures are interesting for studying the interplay between resonant tunneling and the Coulomb blockade effect in single electron structures [84]-[86]. These studies may produce interesting and promising device properties for future electronics, but many practical difficulties due to their extremely small size and increased complexity have to be resolved.

\section{Other NDR Quantum Devices}

We now make a comparison between RTD's and other quantum devices with NDR characteristics. RTT's and RHET's, being three-terminal devices, have the advantage that the NDR can be controlled by the voltage applied to the third terminal, an attractive feature for circuit design, since it provides isolation between different circuit stages. This allows better control of device operation and gain. Although RTT operation has been demonstrated [87], it is extremely difficult to make a low resistive contact to the RTT base. On the other hand, RHET's have limited gain due to scattering in the base and are difficult to fabricate [88]. However, this controllable NDR characteristic can be obtained with RTD's alone by connecting it in series with an FET or an HBT.

Another device whose operation is based on electron tunneling, the Esaki or tunnel diode, is the original quantum device with an NDR I-V curve. At that time, only discrete devices were possible. Now, there is no reason that they cannot also be integrated with HBT's or HEMT's. The challenges are different from those for RTD's in that it is the doping that varies instead of the composition. One or the other may be more appropriate for a given application.

Some of the important features of RTD's, RITD's, and Esaki TD's that have been discussed are summarized in Table 2. A comprehensive comparison among these devices with an in-depth analysis is of current interest, and research is ongoing on both device and circuit aspects. 
Table 2 Important Parameters of RTD's, RITD's, and TD's. Note That at the Swing Voltage, the Device Current in the Second PDR Region Reaches the Peak Current (See Also Fig. 10). The Speed Index Is the Ratio of the Peak Current over the Device Capacitance

\begin{tabular}{|c|c|c|c|}
\hline$\overline{\text { NDR devices }}$ & RTDs & RITDs & TDs \\
\hline Device structure & 1)BQW & polytype QW & $\mathrm{P}^{+} \mathrm{N}^{+}$junction \\
\hline $\begin{array}{l}\text { Operating } \\
\text { principle }\end{array}$ & resonant tunneling & resonant tunneling & $\begin{array}{l}\text { single barrier } \\
\text { tunneling }\end{array}$ \\
\hline Carrier transport & unipolar & bipolar & bipolar \\
\hline Tunneling process & intraband & interband & interband \\
\hline $\begin{array}{l}\mathrm{I}-\mathrm{V} \\
\text { characteristics }\end{array}$ & N-shape NDR & N-shape NDR & $\mathrm{N}$-shape NDR \\
\hline $\begin{array}{l}\text { Peak current } \\
\text { density }\left(\mathrm{A} / \mathrm{cm}^{2}\right)\end{array}$ & high $\left(10^{4}-10^{5}\right)$ & medium $\left(\sim 10^{3}\right)$ & medium $\left(\sim 10^{3}\right)$ \\
\hline $\begin{array}{l}\text { Pcak } \\
\text { voltage }\end{array}$ & small to large & small to large & smiall \\
\hline $\begin{array}{l}\text { Swing } \\
\text { voltage }\end{array}$ & can be large & can be large & matcrial limited \\
\hline $\begin{array}{l}\text { Speed index } \\
\left(\mathrm{mA} / \mathrm{pF}^{\mathrm{H}}\right)\end{array}$ & as high as $10^{3}$ & medium $(\leq 100)$ & medium $(\leq 100)$ \\
\hline $\begin{array}{l}\text { Design } \\
\text { flexibility }\end{array}$ & very good & material limited & $\begin{array}{l}\text { matcrial and doping } \\
\text { limited }\end{array}$ \\
\hline $\begin{array}{l}\text { Manufacture } \\
\text { ability }\end{array}$ & good & difficult & fair \\
\hline $\begin{array}{l}\text { Si technology } \\
\text { compatibility }\end{array}$ & $\begin{array}{l}\text { yes. SiGeC and } \\
\text { other materials }\end{array}$ & $\begin{array}{l}\text { difficult } \\
\text { difficult }\end{array}$ & $\begin{array}{l}\text { yes. SiGeC and } \\
\text { other materials }\end{array}$ \\
\hline
\end{tabular}

\section{CONCLUSIONS}

It is generally agreed that the continued miniaturization of transistors and IC's may open a revolutionary regime for novel semiconductor devices and circuits. Research in nanoelectronics today stands at a frontier of semiconductor science and engineering. The RTD is considered to be a major nanoelectronic device at the center of nanoelectronic research. In this review, we have addressed the following questions. How well do we understand RTD device operation? How accurately are we able to model its $\mathrm{I}-\mathrm{V}$ characteristics and other properties? What basic requirements are there for application in digital logic in terms of the dc I-V characteristics? How realistic it is that the desired $\mathrm{I}-\mathrm{V}$ characteristics can be attained with available material and device structures? These issues were discussed, and recent accomplishments as well as impediments were reviewed.

Numerical modeling is playing an increasingly important role as a tool for gaining insight into nanoelectronic device operation and providing guidance for device design. Quantum transport modeling and computer simulation tools will be very valuable for conceiving new device concepts and exploring novel nanostructures. Sophisticated RTD models have been developed with varying levels of success in reproducing and predicting the $\mathrm{I}-\mathrm{V}$ characteristics. Accurate predictions of the $\mathrm{I}-\mathrm{V}$ characteristics for different material systems and device structures are difficult to achieve. This is due to uncertainties in material growth and device process data as well as in the material parameters, especially when complicated materials and structures are involved. Therefore, more accurate characterization and calculation of material and structural parameters are needed for further development. We also note that RTD models, including multiband and scattering effects, can become quite computationally expensive and demand significant human and computer resources, including a good understanding of advanced quantum physics. Tradeoffs between modeling cost and accuracy should be considered for specific applications.

RTD's are very promising nanoelectronic devices for digital logic circuit application due to their intrinsically high-speed NDR characteristics at room temperatures. Integrated circuits made from the III-V semiconductors are the most likely opportunity for technology insertion, but if a material system compatible with silicon technology could be achieved, the circuits could also be developed for silicon very large scale integration. Another concern for practical RTD applications is that achieving uniformity in resonant tunneling current, which depends exponentially on the parameters of the tunneling barriers, presents a serious challenge for circuit design and performance. However, recent progress indicates that in situ probing of the epitaxial growth, with real-time feedback into the growth process, may adequately achieve this goal. Nanoscience and nanotechnology are broad fields, highly interdisciplinary in breadth and depth. It may take years for RTD's to become routine workhorses in future electronics. Whether or not RTD's will achieve their promise may also be aided by advances in other areas of quantum devices, such as single electronic devices and quantum MOS transistors now under active research.

\section{ACKNOWLEDGMENT}

The authors wish to extend their sincere gratitude to many colleagues for fruitful interactions. Special thanks are due to Dr. H. Grubin of Scientific Research Associates, Dr. W. R. Frensley of the University of Texas at Dallas, Dr. D. K. Ferry of Arizona State University, Dr. G. Klimeck and Dr. R. Lake of Texas Instruments Incorporated, Dr. R. K. Mains and Dr. J. R. East of the University of Michigan, and Dr. Y. Zohta of Tokyo Engineering University for their various contributions to this paper. They also wish to express their true appreciation to the reviewers for their encouragement and constructive criticisms.

\section{REFERENCES}

[1] L. Esaki and R. Tsu, "Superlattics and negative differential conductivity in semiconductors," IBM J. Res. Develop., vol. 14, pp. 61-65, Jan. 1970; R. Tsu and L. Esaki, "Tunneling in a finite superlattice," Appl. Phys. Lett., vol. 22, pp. 562-564, June 1973; L. L. Chang, L. Esaki, and R. Tsu, "Resonant tunneling in semiconductor double barriers," Appl. Phys. Lett., vol. 24, pp. 593-595, June 1974.

[2] P. Mazumder, S. Kulkarni, M. Bhattacharya, J. P. Sun, and G. I. Haddad, "Digital circuit applications of resonant tunneling devices," this issue, pp. 664-686.

[3] T. Murotani, I. Naritake, T. Matano, T. Ohtsuki, N. Kasai, H. Koga, K. Koyama, K. Nakajima, H. Yamaguchi, H. Watanabe, and T. Okuda, "A 4-level storage 4Gb DRAM," in 1997 IEEE Int. Solid-State Circuits Conf. Dig., San Francisco, CA, Feb. 1997, p. 74; and H. Yasuda, "Multimedia impact on devices in the 21st century," in 1997 IEEE Int. Solid-State Circuits Conf. Dig., San Francisco, CA, Feb. 1997, p. 28.

[4] D. V. Averin and K. K. Likharev, "Single electronics: A correlated transfer of single electron and Cooper pairs in systems of small tunnel junctions," in Mesoscopic Phenomena in Solids, B. L. Altshuler, P. A. Lee, and R. A. Webb, Eds. New York: North-Holland, 1991, ch. 6, pp. 173-271. 
[5] D. Goldhaber-Gordon, M. S. Montemerlo, J. C. Love, G. J. Optiteck, and J. Ellenbogen, "Overview of nanoelectronic devices," Proc. IEEE, vol. 85, pp. 521-540, Apr. 1997.

[6] D. A. Allee, A. N. Broers, and R. F. W. Pease, "Limits of nano-gate fabrication," Proc. IEEE, vol. 79, pp. 1093-1105, Aug. 1991; and H. Ahmed, "Nanostructure fabrication," Proc. IEEE, vol. 79, pp. 1140-1148, Aug. 1991.

[7] H. Chan, S. Mohan, P. Mazumder, and G. I. Haddad, "Compact multiple-valued multiplexers using negative differential resistance devices," IEEE J. Solid-State Circuits, vol. 31, pp. 1151-1156, Aug. 1996.

[8] J. van der Wagt, A. Seabaugh, and E. A. Beam III, "RTD/HFET low standby power SRAM gain cell," in IEDM 1996 Technical Digest. New York: IEEE Press, 1996, pp. 425-428.

[9] W. Williamson III, S. B. Enquist, D. H. Chow, H. L. Dunlap, S. Subramaniam, P. M. Lei, G. H. Bernstein, and B. K. Gilbert, "12 $\mathrm{GHz}$ clocked operation of ultra-low power interband resonant tunneling diode pipelined logic gates," IEEE J. Solid-State Circuits, vol. 32, pp. 222-231, Feb. 1997.

[10] T. Waho, K. J. Chen, and M. Yamamoto, "Novel multiplevalued logic gate using resonant tunneling devices," IEEE Electron Device Lett., vol. 17, pp. 223-225, May 1996.

[11] S. Luryi, "Frequency limit of double barrier resonant tunneling oscillators," Appl. Phys. Lett., vol. 47, pp. 490-492, Sept. 1985.

[12] W. R. Frensley, "Boundary conditions for open quantum systems driven far from equilibrium," Rev. Mod. Phys., vol. 62, pp. 745-791, July 1990.

[13] D. K. Ferry and H. L. Grubin, "Modeling of quantum transport in semiconductor devices," in Solid State Physics, Advances in Research and Applications, vol. 49, H. Ehrenreich and F. Spaepen Eds. New York: Academic, 1995, pp. 283-448.

[14] M. Altarelli, "Band structure, impurities and excitons in superlattices," in Heterostructures and Semiconductor Superlattices, G. Allan and G. Bastard, Eds. Berlin: Springer-Verlag, 1996, pp. 12-37; and G. Bastard, J. A. Brum, and R. Ferreira, "Electron states in semiconductor heterostructures," in Solid State Physics, Advances in Research and Applications, vol. 44, H. Ehrenreich and D. Turnbull, Eds. New York: Academic, 1991, pp. 229-415.

[15] R. Landauer, "Electrical resistance of disordered onedimensional lattices," Philosophy Mag., vol. 21, pp. 863-867, 1970.

[16] M. Büttiker, Y. Imry, R. Landauer, and S. Pinhas, "Generalized many-channel conductance formula with applications to small rings," Phys. Rev. B., vol. 31, pp. 6207-6215, May 1985.

[17] R. Tsu and L. Esaki, "Tunneling in a finite superlattice," Appl. Phys. Lett., vol. 22, pp. 562-564, June 1973.

[18] H. Ohnishi, T. Inata, S. Muto, N. Yokoyama, and A. Shibatomi, "Self-consistent analysis of resonant tunneling devices," Appl. Phys. Lett., vol. 49, pp. 1248-1250, Nov. 1986.

[19] M. Cahay, M. Mclennan, S. Datta, and M. S. Lundstrom, "Importance of space charge effects in resonant tunneling devices," Appl. Phys. Lett., vol. 50, pp. 612-614, Mar. 1987.

[20] J. P. Sun, R. K. Mains, W. L Chen, J. R. East, and G. I. Haddad, "C-V and I-V characteristics quantum well varactors," J. Appl. Phys., vol. 72, pp. 2340-2346, Sept. 1992

[21] T. Fiig and A. P. Jauho, "Self-consistent model for twodimensional accumulation layer states in resonant tunneling devices," Appl. Phys. Lett., vol. 59, pp. 2245-2247, Oct. 1991.

[22] E. O. Kane, "The k · p method," in Semiconductors and Semimetals, vol. 1, R. K. Willardson and A. C. Beer, Eds. New York: Academic, 1966, pp. 75-100.

[23] J. N. Schulman, "Ga $\mathrm{Ga}_{-x} \mathrm{Al}_{x} \mathrm{As}-\mathrm{Ga}_{1-y} \mathrm{Al}_{y} \mathrm{As}-\mathrm{GaAs}$ doublebarrier structures," J. Appl. Phys., vol. 60, pp. 3954-3958, Dec. 1986

[24] K. V. Rousseau, K. L. Wang, and J. N. Schulman, " $\Gamma$ and $X$ state influence on resonant tunneling current in single and double barrier GaAs/AlAs structures," Appl. Phys. Lett., vol. 54, pp. 1341-1343, Apr. 1989.

[25] P. Vogl, H. P. Hjalmarson, and J. D. Dow, "A semi-empirical tight-binding theory of the electronic structure of semiconductors," J. Phys. Chem. Solids, vol. 44, pp. 365-378, May 1983

[26] T. B. Boykin, "Incorporation of incompleteness in the $\mathrm{k} \cdot \mathrm{p}$ perturbation theory," Phys. Rev. B, vol. 52, pp. 16317-16320, Dec. 1995.

[27] R. C. Bowen, G. Klimeck, R. K. Lake, W. R. Frensley, and T. Moise, "Quantitative simulation of a resonant tunneling diode,"
J. Appl. Phys., vol. 81, pp. 3207-3213, Apr. 1997.

[28] F. Chevoir and B. Vinter, "Calculation of phononassisted tunneling and valley current in a double-barrier diode," Appl. Phys. Lett., vol. 55, pp. 1859-1861, Oct. 1989.

[29] P. J. Turley and S. W. Teitsworth, "Theory of localized phonon modes and their effects ion electron tunneling in double barrier structures," J. Appl. Phys., vol. 72, pp. 2356-2366, Sept. 1992.

[30] P. Roblin and W. R. Liou, "Three-dimensional scatteringassisted tunneling in resonant-tunneling diodes," Phys. Rev. B, vol. 47, pp. 2146-2161, Jan. 1993.

[31] W. Cai, T. F. Zheng, P. Hu, B. Yudanin, and M. Lax, "Model of phonon associated electron tunneling through a semiconductor double barrier," Phys. Rev. Lett., vol. 63, pp. 418-421, July 1989.

[32] Y. Fu, Q. Chen, and M. Willander, "Evanescent channels in calculation of phononassisted tunneling spectrum of a semiconductor tunneling structure," J. Appl. Phys., vol. 74, pp. 1848-1852, Feb. 1993

[33] A. D. Stone and P. A. Lee, "Effect of inelastic processes on resonant tunneling in one dimension," Phys. Rev. Lett., vol. 54, pp. 1196-1199, Mar. 1985.

[34] Y. Zohta and T. Tanamoto, "Improved optical model for resonant tunneling diode," J. Appl. Phys., vol. 74, pp. 6996-6998, Dec. 1994.

[35] A. Bohr and B. Mottelson, Nuclear Structure, vol. 1. New York: Benjiamin, 1969

[36] J. P. Sun and G. I. Haddad, "Self-consistent scattering calculation of resonant tunneling diode characteristics," presented at the 1995 International Workshop for Computational Electronics, Tempe, AZ, Oct. 1995.

[37] W. R. Frensley, "Wigner function model of a resonant tunneling device," Phys. Rev. B, vol. 36, pp. 1570-1580, July 1987.

[38] R. K. Mains and G. I. Haddad, "Wigner function modeling of resonant tunneling diodes with high peak-to-valley ratios," $J$. Appl. Phys., vol. 64, pp. 5041-5044, Nov. 1988.

[39] K. L. Jensen and F. A. Buot, "The effects of scattering on current-voltage characteristics, transient response, and particle trajectories in the numerical simulation of resonant tunneling diodes," J. Appl. Phys., vol. 67, pp. 7602-7607, June 1990.

[40] N. C. Kluksdahl, A. M. Kriman, C. Ringhofer, and D. K. Ferry, "Quantum tunneling properties from a Wigner function study," Solid-State Electron., vol. 31, pp. 743-746. Mar./Apr. 1988.

[41] R. K. Mains and G. I. Haddad, "An accurate re-formulation of the Wigner function method for quantum transport modeling," J. Comput. Phys., vol. 112, pp. 149-161, May 1994.

[42] H. L. Grubin, "Density matrix simulations of semiconductor devices," in Quantum Transport in Ultra-Small Devices, D. K. Ferry, H. L. Grubin, C. Jacoboni, and A. Jauho, Eds., NATO ASI Series B: Physics. New York: Plenum, 1995, vol. 342, p. 241

[43] F. A. Buot, "Mesoscopic physics and nanoelectronics: Nanoscience and nanotechnology," Phys. Rep., vol. 234, pp. 73-174, Nov. 1993

[44] E. V. Anda and F. Flores, "The role of inelastic scattering in resonant tunneling heterostructures," J. Phys. Condens. Matter, vol. 3, pp. 9087-9101, Nov. 1991

[45] R. Lake and S. Datta, "Nonequilibrium Green's function method applied to double barrier resonant tunneling diodes," Phys. Rev. $B$, vol. 45, pp. 6670-6685, Mar. 1992.

[46] G. Klimeck, R. Lake, R. C. Bowen, W. R. Frensley, and D. Blanks, "NanoElectronic MOdeling (NEMO)," in 53rd Annual Device Research Conference Digest. New York: IEEE Press, 1995, pp. 52-53; R. Lake, G. Klimeck, R. C. Bowen, C. Fernando, D. Jovanovic, D. Blanks, T. S. Moise, M. Leng, and W. R. Frensley, "Experimentally verified quantum device simulations based on multiband models, Hartree selfconsistency, and scattering assisted charging," in 54th Annual Device Research Conference Digest. New York: IEEE Press, 1996, pp. 174-175.

[47] E. N. Economou, Green's Functions in Quantum Physics, Springer Series in Solid-State Science, vol. 7. Berlin: Springer-Verlag, 1983.

[48] L. V. Keldysh, "Diagram technique for nonequilibrium processes," Sov. Phys. JETP, vol. 20, pp. 1018-1026, Apr. 1965.

[49] L. P. Kadanoff and G. Baym, Quantum Statistical Mechanics. NY: Addison-Wesley, 1989.

[50] S. Datta, Electronic Transport in Mesoscopic Systems. Cambridge, U.K.: Cambridge Univ. Press, 1995.

[51] G. D. Mahan, "Quantum transport equation for electric and 
magnetic fields," Phys. Rep., vol. 145, pp. 251-318, Jan. 1987

[52] S. Datta, "Steady state quantum kinetic equation," Phys. Rev. $B$, vol. 40, pp. 5830-5833, Sept. 1989.

[53] N. S. Wingreen, A. Jauho, and Y. Meir, "Time-dependent transport through a mesoscopic structure," Phys. Rev. B, vol. 48, pp. 8487-8490, Sept. 1993.

[54] A. Jauho, N. S. Wingreen, and Y. Meir, "Time-dependent transport in interacting and noninteracting resonant-tunneling systems," Phys. Rev. B, vol. 50, pp. 5528-5544, Aug. 1994.

[55] C. S. Lent and D. J. Kirkner, "The quantum transmitting boundary method," J. Appl. Phys., vol. 67, pp. 6353-6359, May 1990.

[56] J. P. Sun, R. K. Mains, K. Yang, and G. I. Haddad, "A self-consistent model of $\Gamma-X$ mixing in GaAs/AlAs/GaAs quantum well structures using the quantum transmitting boundary method," J. Appl. Phys., vol. 74, pp. 5053-5060, Oct. 1993.

[57] D. D. Coon and H. C. Liu, "Frequency limit of double barrier resonant tunneling oscillators," Appl. Phys. Lett., vol. 49, pp. 94-96, July 1986.

[58] C. E. Chang, P. M. Asbeck, K. C. Wang, and E. R. Brown, "Analysis of heterojunction bipolar transistor/resonant tunneling diode logic for low-power and high-speed digital applications," IEEE Trans. Electron Devices, vol. 40, pp. 685-691, Apr. 1993

[59] S. Mohan, J. P. Sun, P. Mazumder, and G. I. Haddad, "Device and circuit simulation of quantum electronic devices," IEEE Trans. Computer-Aided Design, vol. 14, pp. 653-662, June 1995.

[60] J. N. Schulman, H. J. De Los Santos, and D. H. Chow, "Physicsbased RTD current-voltage equation," IEEE Electron Device Lett., vol. 17, pp. 220-222, May 1996.

[61] P. Huang, D. S. Pan, and N. C. Luhmann, Jr. "A microwave measurement technique for characterizing the I-V relationship for negative differential conductance devices," IEEE Trans. Microwave Theory Tech., vol. 41, pp. 1455-1458, Aug. 1993.

[62] H. C. Liu and T. C. L. G. Sollner, "High-frequency resonant tunneling diodes," in Semiconductors and Semimetals, vol. 41, High-Speed Heterostructure Devices, R. A. Kiehl and T. C. L. G. Sollner, Eds. New York: Academic, 1994, pp. 359-419.

[63] E. R. Brown, J. R. Soderstrom, C. D. Parker, L. J. Mahoney, K. M. Molvar, and T. C. McGill, "Oscillation up to $712 \mathrm{GHz}$ in InAs/AlSb resonant tunneling diodes," Appl. Phys. Lett., vol. 58, pp. 2291-2293, May 1991.

[64] A. Gruhle, H. Kibbel, U. Erben, and E. Kasper, "91 GHz SiGe HBT's grown by MBE," Electron. Lett., vol. 29, pp. 415-417, Feb. 1993.

[65] K. Ismail, B. S. Meyerson, and P. J. Wang, "Electron resonant tunneling in SiGe double barrier diodes," Appl. Phys. Lett., vol. 59, pp. 973-975, Aug. 1991.

[66] U. Konig, M. Kuisl, J. F. Luy, and F. Schaffler, "Si/SiGe resonant tunneling devices separated by surrounding polysilicon," Electron. Lett., vol. 26, pp. 1904-1906, 1990.

[67] H. C. Liu, D. Landheer, M. Buchanan, and D. C. Houghton, "Resonant tunneling in $\mathrm{Si} / \mathrm{Si}_{1-x} \mathrm{Ge}_{x}$ double barrier structures," Appl. Phys. Lett., vol. 52, pp. 1809-1811, May 1988.

[68] U. Gennser, V. P. Kesan, S. S. Iyer, T. J. Bucelot, and E. S Yang, "Resonant tunneling of holes through silicon barriers," $J$. Vac. Sci. Technol., vol. 8, pp. 210-213, Mar./Apr. 1990.

[69] R. Tsu, "Silicon-based quantum wells," Nature, vol. 364, p. 19 July 1993.

[70] R. A. Soref, "Silicon-based optoelectronics," Proc. IEEE, vol. 81, pp. 1687-1706, Dec. 1993.

[71] A. Seabaugh and R. Lake, "Tunnel diodes," in Encyclopedia of Applied Physics, vol. 20. New York: VCH, 1996.

[72] T. Broekaert, W. Lee, and C. G. Fonstad, "Pseudomorphic $\mathrm{In}_{.53} \mathrm{Ga}_{.47} \mathrm{As} / \mathrm{AlAs} / \mathrm{InAs}$ resonant tunneling diodes with peakto-valley ratios of 30 at room temperature," Appl. Phys. Lett., vol. 53, pp. 1545-1547, Oct. 1988.

[73] J. R. Soderstrom, D. H. Chow, and T. C. McGill, "New negative differential resistance device based on resonant interband tunneling," Appl. Phys. Lett., vol. 55, pp. 1094-1096, Sept. 1989.

[74] L. F. Luo, B. Beresford, and W. I. Wang, "Interband tunneling in polytype GaSb/AlSb/InAs heterostructures," Appl. Phys. Lett., vol. 55, pp. 2023-2025, Nov. 1989.

[75] M. Sweeny and J. M. Xu, "Resonant interband tunnel diodes," Appl. Phys. Lett., vol. 54, pp. 546-548, Feb. 1989.

[76] M. Reddy, M. J. Mondry, M. J. W. Rodwell, S. C. Martin, R. P. Smith, D. H. Chow, and J. N. Schulman, "Fabrication and dc, microwave characteristics of submicron Schottky-collector $\mathrm{AlAs} / \mathrm{In}{ }_{53} \mathrm{Ga}_{47} \mathrm{As} / \mathrm{InP}$ resonant tunneling diodes," J. Appl. Phys., vol. 77, pp. 4819-4821, May 1995.

[77] T. Nakagawa, H. Inamoto, T. Kojoima, and K. Ohta, "Observation of resonant tunneling in $\mathrm{AlGaAs} / \mathrm{GaAs}$ triple barrier diodes," Appl. Phys. Lett., vol. 49, pp. 73-75, July 1986.

[78] H. Mizuta, T. Tanoue, and S. Takahashi, "A new triple-well resonant tunneling diode with controllable double-negative resistance," IEEE Trans. Electron Devices, vol. ED-35, pp. 1951-1956, Nov. 1988.

[79] A. Kumar and P. H. Bagwell, "Resonant tunneling in a quasione-dimensional wire: Influence of evanescent modes," Phys. Rev. B, vol. 43, pp. 9012-9020, Apr. 1991.

[80] M. A. Reed, J. N. Randall, R. J. Aggarwal, R. J. Matyi, T. M Moore, and A. E. Westel, "Observation of discrete electronic states in zero-dimensional semiconductor nanostructure," Phys. Rev. Lett., vol. 60, pp. 535-537, Feb. 1988

[81] D. Day, Y. Chung, C. Webb, J. N. Eckstein, J. M. Xu, and M. Sweeny, "Heterostructure p-n junction tunnel diodes," Appl. Phys. Lett., vol. 57, pp. 1140-1142, Sept. 1990.

[82] M. Reddy, S. C. Martin, A. C. Molnar, R. E. Muller, R. P Smith, P. H. Siegel, M. J. Mondry, M. J. W. Rodwell, and S. J. Allen, Jr., "Monolithic Schottky-collector resonant tunnel diode oscillator arrays to $650 \mathrm{GHz}$," IEEE Electron Device Lett., vol. 18, pp. 218-221, May 1997

[83] C. J. Goodings, H. Mizuta, J. R. A. Cleaver, and H. Ahmed, "Variable-area resonant tunneling diodes using implanted inplane gates," J. Appl. Phys., vol. 76, pp. 1276-1286, July 1994.

[84] M. Tewordt, L. Martin-Moreno, T. J. Nicholls, M. Pepper, M. J. Kelly, V. J. Law, D. A. Ritchie, J. E. F. Frost, and G. A. C. Jones, "Single electron tunneling and Coulomb charging effects in asymmetric double-barrier resonant tunneling diodes," Phys. Rev. B, vol. 45, pp. 14407-14410, June 1992.

[85] M. W. Dellow, C. J. G. M. Langerak, P. H. Beton, T. J. Foster, P. C. Main, L. Eaves, M. Henini, S. P. Beaumont, and C. D. W. Wilkinson, "Single electron tunneling through a donor state in a gated resonant tunneling device," Surface Sci., vol. 263 , pp. 438-441, Feb. 1992.

[86] B. Su, V. J. Goldman, and J. E. Cunningham, "Single-electron tunneling in nanometer-scale double-barrier heterostructure devices," Phys. Rev. B, vol. 46, pp. 7644-7655, Sept. 1992

[87] G. I. Haddad, U. K. Reddy, J. P. Sun, and R. K. Mains, "The bound-state resonant tunneling transistor (BSRTT): Fabrication, d.c. I-V characteristics, and high-frequency properties," $\mathrm{Su}$ perlattices and Microstructures, vol. 7, no. 4, pp. 369-374, 1990.

[88] N. Yokoyama, H. Ohnishi, T. Futatsugi, S. Muto, T. Mori, K. Imamura, and A. Shibatomi, "Resonant-tunneling transistors using InGaAs-based materials," Proc. SPIE, vol. 943, pp. 14-21, Mar. 1988.

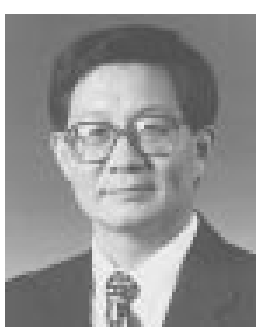

Jian Ping Sun received the B.S. degree from Nanjing Institute of Technology, China, and the M.S. and Ph.D. degrees from the University of Michigan, Ann Arbor, all in electrical engineering.

He was with the Nanjing Solid State Devices Research Institute, China, and the Electronics Research Laboratories, University of California at Berkeley. He is with the Solid State Electronics Laboratory, Department of Electrical Engineering and Computer Science, University of Michigan, where he is an Assistant Research Scientist and Visiting Assistant Professor. His research interests include modeling and design of high-speed, high-frequency semiconductor devices, quantum devices, and photonic devices. 


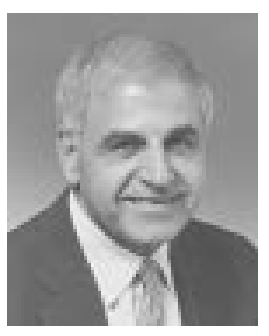

George I. Haddad (Life Fellow, IEEE) received the B.S.E., M.S.E., and Ph.D. degrees in electrical engineering from the University of Michigan, Ann Arbor.

In 1958, he joined the Electron Physics Laboratory, where he engaged in research on masers, parametric amplifiers, detectors, and electronbeam devices. From 1960 to 1969 , he was successively Instructor, Assistant Professor, Associate Professor, and Professor in the Electrical Engineering Department of the University of Michigan. In 1991, he was named the Robert J. Hiller Professor of Electrical Engineering. During 1975-1987 and 1988-1997, he was Chairman of the Department of Electrical Engineering and Computer Science. He currently is Director of the Center for High-Frequency Microelectronics. His current research areas are microwave and millimeter-wave solid-state devices, monolithic integrated circuits, and microwave-optical interactions.

Dr. Haddad is a member of Eta Kappa Nu, Sigma Xi, Phi Kappa Phi, Tau Beta Pi, and the American Society for Engineering Education. He was Editor of IEEE TRANSACtions ON Microwave THEORY AND TeChNIQUES from 1968 to 1971. He received the 1970 Curtis W. McGraw Research Award from the American Society for Engineering Education for outstanding achievements by an engineering teacher, the College of Engineering Excellence in Research Award (1985), the Distinguished Faculty Achievement Award (1986), and the S. S. Atwood Award for excellence in engineering research, education, and administration.

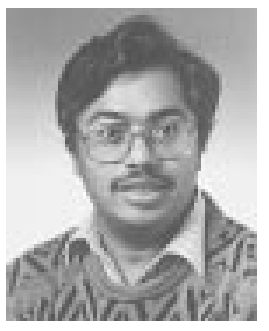

Pinaki Mazumder (Senior Member, IEEE) received the B.S.E.E. degree from the Indian Institute of Science in 1976, the M.Sc. degree in computer science from the University of Alberta, Canada, in 1985, and the Ph.D. degree in electrical and computer engineering from the University of Illinois at Urbana-Champaign in 1987.

For two years, he was a Research Assistant with the Coordinated Science Laboratory, University of Illinois at Urbana-Champaign. For more than six years, he was with Bharat Electronics, Ltd., India, where he developed analog and digital integrated circuits for consumer electronics products. During the summers of 1985 and 1986, he was a Member of the Technical Staff in the Naperville branch of AT\&T Bell Laboratories. He presently is an Associate Professor with the Department of Electrical Engineering and Computer Science, University of Michigan, Ann Arbor. His research interests include very-large-scale-integration testing, physical design automation, ultrafast digital circuit design, and neural hardware.

Dr. Mazumder is a member of Sigma Xi, Phi Kappa Phi, and the Association of Computing Machinery Special Interest Group on Design Automation.

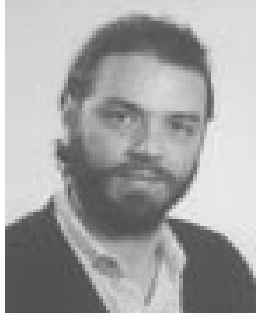

Joel N. Schulman received the B.A. degree in physics from the University of Pennsylvania, Philadelphia, in 1973 and the Ph.D. degree in physics from the California Institute of Technology, Pasadena, in 1979.

$\mathrm{He}$ is a Senior Research Scientist in the Sensors and Materials Laboratory of HRL Laboratories, Malibu, CA, and an Adjunct Professor in the Department of Electrical Engineering at the University of California, Los Angeles. His research interests include the electronic and optical properties of semiconductor superlattices, quantum wells, and tunnel structures. 